\title{
Polycyclic aromatic hydrocarbon selected galaxies ${ }^{\star}$
}

\author{
M. Haas ${ }^{1}$, C. Leipski ${ }^{2}$, R. Siebenmorgen ${ }^{3}$, H. Meusinger ${ }^{4}$, H. Drass ${ }^{1}$, and R. Chini ${ }^{1}$ \\ 1 Astronomisches Institut Ruhr-Universität Bochum, Universitätsstraße 150, 44801 Bochum, Germany \\ e-mail: haas@astro.rub.de \\ 2 Department of Physics, University of California, Santa Barbara, CA 93106, USA \\ 3 European Southern Observatory, Karl-Schwarzschild-Str. 2, 85748 Garching, Germany \\ 4 Thüringer Landessternwarte Tautenburg, Sternwarte 5, 07778 Tautenburg, Germany
}

Received 16 June 2009 / Accepted 5 August 2009

ABSTRACT

\begin{abstract}
Context. This is the fourth in a series of papers based on the ISOCAM Parallel Survey at $6.7 \mu \mathrm{m}$. While the first three papers have been devoted to active galactic nuclei (AGN), here we report on emission-line galaxies without AGN signatures in their optical spectra. Aims. Polycyclic aromatic hydrocarbon (PAH) emission has been found in both starbursts and modestly starforming galaxies, but the relation between starforming activity and PAH luminosity is still a matter of debate. The different correlation degrees could be caused by the variety of optical and far-infrared sample selection criteria. In order to obtain a census of the typical properties of PAH emitting galaxies, we here study moderately distant galaxies which have been selected by their PAH emission.

Methods. Combining the ISOCAM Parallel Survey at $6.7 \mu \mathrm{m}$ with 2MASS we have colour-selected a sample of 120 candidates for strong PAH emission. We obtained optical and mid-infrared spectra of 75 and 19 sources, respectively, and analysed IRASADDSCANs and available Spitzer 3.6-160 $\mu$ m photometry.

Results. The Spitzer mid-infrared spectra exhibit clear PAH features and corroborate that our photometric selection criteria trace the PAH emission of galaxies fairly well. The optical spectra show emission lines, at median redshift $z \sim 0.1$, as well as $\mathrm{H}_{\delta}$ and $\mathrm{Ca}$ II absorption, indicating ongoing starformation as well as post-starbursts. The mid- and far-infrared spectral energy distributions (SEDs) provide evidence for a broad range of far-infrared (FIR) luminosities $\left(10^{9} L_{\odot}-2 \times 10^{12} L_{\odot}\right)$, but in general the dust is colder $(T \lesssim 25 \mathrm{~K}$, $\beta=2)$ than for starburst galaxies like $\mathrm{M} 82(T \approx 40 \mathrm{~K})$. For most galaxies the monocromatic luminosity $\left(v \cdot L_{v}\right)$ peaks at about equal height at optical, $6.7 \mu \mathrm{m}$ (PAH) and FIR wavelengths. In about 15\% of the sources the FIR luminosity exceeds the optical and PAH energy output by about a factor 5-10 despite the cool dust temperature; in these galaxies a large dust mass of $10^{8}-10^{9} M_{\odot}$ is inferred. Conclusions. At moderate distance $(z \sim 0.1)$, PAH selected galaxies turn out to be a quite heterogeneous population of dust-rich, partly infrared-luminous galaxies but mostly cool with a range of post-starburst signatures and starforming activity which appears to be rather modest relative to the entire gas content (derived from the dust mass and assuming a standard gas/dust ratio). Our results on PAH selected galaxies question the often expressed interpretation that the majority of high redshift galaxies detected in 15 and $24 \mu \mathrm{m}$ surveys are dominated by powerful ongoing starbursts with high starforming efficiency.
\end{abstract}

Key words. galaxies: active - infrared: galaxies

\section{Introduction}

Polycyclic aromatic hydrocarbonates (PAHs) emit prominent features in the mid-infrared around 6-9 $\mu \mathrm{m}$ (Puget \& Leger 1989). PAH carriers are widely distributed in the dusty interstellar medium of our Galaxy (Mattila et al. 1996) and they are excited in the photodissociation regions (Cesarsky et al. 1996b) as well as by the mild UV-radiation field around A- and F-type stars (Lemke et al. 1998; Uchida et al. 2000). PAH emission occurs in normal spirals (e.g. Mattila et al. 1999) as well as in luminous and ultra-luminous starbursts (ULIRGs, Genzel et al. 1998). PAH features have been detected also in dusty elliptical galaxies (Bregman et al. 2008; Kaneda et al. 2008) and in AGN (Schweitzer et al. 2006; Shi et al. 2007), although faint above the underlying continuum.

In the local universe $(z<0.01)$, the PAH flux appears to be better correlated with the 850 and $160 \mu \mathrm{m}$ emission from cold dust than with the 15 and $24 \mu \mathrm{m}$ warm dust emission (Haas et al. 2002; Bendo et al. 2008). Compared with normal galaxies, ULIRGs show an enhanced $100 \mu \mathrm{m} / \mathrm{PAH}$ flux ratio

* Tables 1-3 are only available in electronic form at http://www . aanda.org
(Klaas et al. 2001), and among optically selected metal-rich starforming galaxies the $70 \mu \mathrm{m} / 8 \mu \mathrm{m}$ flux ratio increases with luminosity (Monkiewicz et al. 2008). An analysis of Revised Shapley-Ames galaxies in the SINGS survey suggests that it is uncertain by a factor of 10 to use the PAH luminosity to extrapolate the far-infrared (FIR, $>60 \mu \mathrm{m}$ ) properties and the starforming activity (Dale et al. 2005). For example, consider two galaxies with the same PAH luminosity: A quiescent one like NGC 891 with a large amount of cool dust (SED peaking at $120 \mu \mathrm{m}$, with star forming efficiency $L_{\mathrm{IR}} / M_{\text {gas }} \sim 1 L_{\odot} / M_{\odot}$, Chini et al. 1986) and an active one like M 82 with about a factor of 10 less dust mass which is heated to higher temperatures (SED peaking at $60 \mu \mathrm{m}$, with star forming efficiency $L_{\mathrm{IR}} / M_{\mathrm{gas}} \sim 50 L_{\odot} / M_{\odot}$, Thronson et al. 1987). Despite their similarity in PAH luminosity, the galaxies are clearly different in FIR luminosity and starforming activity, hence different in physical states.

Because the underlying continuum from stars and very small dust grains is relatively weak (except for AGN and elliptical galaxies), the 6-9 $\mu \mathrm{m}$ PAH emission of local galaxies dominates by far $(\gtrsim 80 \%)$ the total flux seen in ISOCAM $6.7 \mu \mathrm{m}$ and Spitzer $8 \mu \mathrm{m}$ broad band images. At cosmological distances the PAH 
emission shifts into the ISOCAM $15 \mu \mathrm{m}(z=1)$ and Spitzer $24 \mu \mathrm{m}(z=2)$ passbands and could dominate the observed fluxes as well. This could naturally explain the peak found in the differential galaxy number counts, without invoking an extraordinary new galaxy population (Xu 2000). While for high-redshift galaxy populations (detected in the GOODS fields in the 15 and $24 \mu \mathrm{m}$ passbands) the rest-frame FIR observations are in general not available, models derived from local - mostly IRAS detected - templates suggest also a high far-infrared luminosity (e.g. Elbaz et al. 2002; Caputi et al. 2007). But the key question remains open as to whether PAH emitting galaxies typically show an intense M 82-like starburst activity, presumably affecting the whole galaxy, or whether the sources are large dust-rich systems with moderate star formation relative to the total gas content. Therefore, it is vital to establish the nature of PAH emitting galaxies over a range of luminosities and distances.

Combining the spectroscopic SDSS galaxy sample with the Spitzer First Look and SWIRE surveys, a good correlation between $8 \mu \mathrm{m}, 24 \mu \mathrm{m}$ and $70 \mu \mathrm{m}$ luminosity has been reported (Wu et al. 2005; Zhu et al. 2008). However, these samples are optically selected to have strong $\mathrm{H}_{\alpha}$ emission and, because SWIRE reaches much deeper than SDSS, a substantial fraction of PAH emitting IR galaxies may have been excluded; for example these samples do not contain any ULIRGs. Therefore, it may be put into question as to how far these correlations from optically selected samples are relevant for properly selected PAH emitting galaxies.

The ISO-ELAIS survey contains some fields observed at $6.7 \mu \mathrm{m}$ (e.g. Väisänen et al. 2002), but so far no specific results on PAH emission have been reported, since the survey focused on exploring the cosmic starforming history to higher redshifts mainly using the 15 and $90 \mu \mathrm{m}$ data (Rowan-Robinson et al. 2004 and references therein). The ISO-FIRBACK survey at $170 \mu \mathrm{m}$ has found a population of IR-luminous but cold galaxies, rather neglected up to now (Patris et al. 2003; Dennefeld et al. 2005). While 22 of these FIR selected galaxies with clean counterparts at shorter wavelengths show PAH emission as inferred from Spitzer-IRAC photometry, the modelled $L_{3-1000 \mu \mathrm{m}} / L_{\mathrm{PAH}}$ ratio in total spreads by about a factor of ten (Sajina et al. 2006).

A proper study of the census of $\mathrm{PAH}$ emitting galaxies should be built on galaxy samples selected by their PAH emission. We here report such a study at moderate distance $(z \sim 0.1)$ based on the ISOCAM $6.7 \mu \mathrm{m}$ Parallel Survey. Distances in this paper are calculated using a $\Lambda$ CDM cosmology with $H_{0}=$ $71 \mathrm{~km} \mathrm{~s}^{-1} \mathrm{Mpc}^{-1}, \Omega_{\mathrm{m}}=0.27$ and $\Omega_{\Lambda}=0.73$.

\section{Sample selection}

\subsection{ISOCAM 6.7 $\mu \mathrm{m}$ Parallel Survey and 2MASS}

The most prominent PAH emission features lie around $7 \mu \mathrm{m}$ (with peaks at 6.2, 7.7 and $8.6 \mu \mathrm{m}$ ) and at $11.3 \mu \mathrm{m}$. The bandpass of the ISOCAM $L W 2$ filter is $5-8.5 \mu \mathrm{m}$ centered at $6.7 \mu \mathrm{m}$. Thus, $L W 2$ is able to measure $6.2 \mu \mathrm{m}$ PAH emission for moderately distant galaxies up redshift $z \sim 0.3$.

Parallel to the observation of a prime target by ISO's photometer ISOPHOT or the spectrographs ISOSWS/ISOLWS, the mid-infrared camera ISOCAM (Cesarsky et al. 1996a) randomly mapped sky locations next to the prime target using the $L W 2$ filter. This results in the $6.7 \mu \mathrm{m}$ ISOCAM Parallel Survey. It covers a total area of $27 \mathrm{deg}^{2}$ and contains about 16000 point sources $\left(F H W M=5^{\prime \prime}\right)$ with a flux limit down to $F(6.7 \mu \mathrm{m}) \sim 1 \mathrm{mJy}$ (Siebenmorgen et al. 1996, 2000; Ott et al. 2003; Ott et al. in preparation). At high galactic latitude $|b|>20^{\circ}$, the surveyed

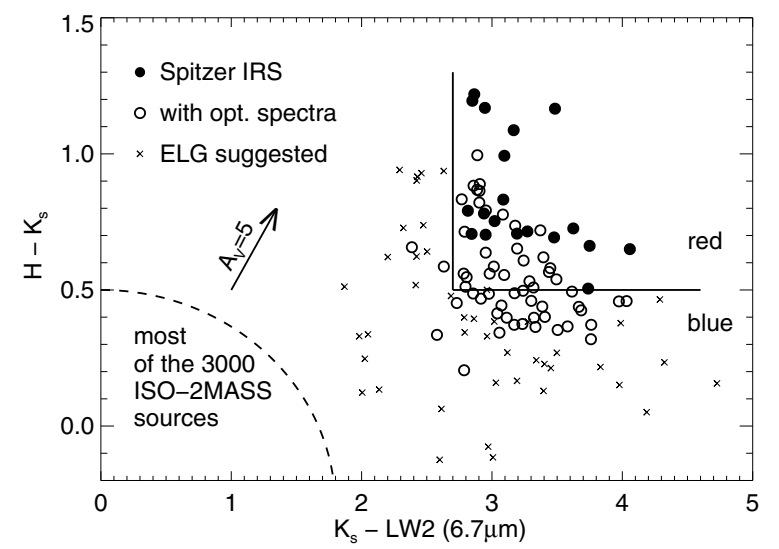

Fig. 1. Colour-colour diagram illustrating the sample selection. While most of the 3000 ISO-2MASS sources lie in the region around zero outlined by the dashed curve, here we consider only sources with $6.7 \mu \mathrm{m}$ excess over $2.2 \mu \mathrm{m}$ emission $\left(K_{\mathrm{s}}-L W 2>8\right)$. We obtained optical spectra for all sources in the upper right corner (red subsample, indicated by the solid lines) and a random subset of the remaining sources. Sources without optical spectra are suggested emission line galaxies (ELGs). Spitzer IRS spectra were taken for the brightest red sources (filled circles). AGN would populate the red colour range; they are not shown here. Local starforming galaxies like NGC 891, NGC 6946, Arp 244 and M 82 have $H-K_{\mathrm{s}}<0.5$ and $K_{\mathrm{s}}-L W 2>2.7$.

area is about $10 \mathrm{deg}^{2}$. It provides a suitable hunting ground to search for galaxies with prominent PAH emission.

In order to select such galaxies, we have matched (within a radius of $2^{\prime \prime}$ ) the ISOCAM sources with the 2MASS point source catalog, with the USNO-B, DSS and UCAC optical catalogues, as well as the NVSS and FIRST radio surveys, and analysed IRAS ADDSCANs. The point source criterion ensures that the galaxies are compact and/or at sufficient distance to be unresolved by these two surveys ( 5 " angular extend corresponds to $\sim 5 \mathrm{kpc}$ at $z=0.1$ ). We excluded objects which have multiple NIR and optical counterparts within $10^{\prime \prime}$, or are contaminated by extended sources (2MASS XSC), or have proper motion ( $p m>3 \sigma$ from UCAC). At $|b|>20^{\circ}$, this yields about 3000 isolated ISO-2MASS sources with clean photometry. The brightness of the sources goes down to $B \sim 20.5 \mathrm{mag}, K_{\mathrm{s}} \sim 15.5 \mathrm{mag}$, $L W 2 \sim 12$ mag (Vega based system). The typical uncertainties on the $H-K_{\mathrm{s}}$ and $K_{\mathrm{s}}-L W 2$ colours are about $0.2 \mathrm{mag}$ and $0.4 \mathrm{mag}$, respectively. Essentially none of the sources is listed in the NVSS and FIRST radio catalogues. Note that our procedure may have excluded closely interacting galaxy pairs.

\subsection{Near-mid-infrared colour selection}

The source selection is described in more detail by Haas et al. (2004, Paper 1). We give a brief desciption here: stars and passive elliptical galaxies populate the colour range $K_{\mathrm{s}}-L W 2<1$. About 150 of the 3000 ISO-2MASS sources show a $6.7 \mu$ m flux excess which we defined by $K_{\mathrm{s}}-L W 2>1.8$ (Fig. 1). We consider them as candidates for galaxies with strong PAH emission (hereafter named PAH-candidates). The threshold $K_{\mathrm{s}}-L W 2>$ 1.8 is based on the comparison with local normal galaxy populations (Dale et al. 2001) as well as luminous and ultra-luminous infrared galaxies (Genzel et al. 1998; Klaas et al. 2001; Laurent et al. 2000) for which we found suitable $L W 2$ data in the ISO Data Archive ${ }^{1}$. While among local galaxies only ultra-luminous infrared galaxies (ULIRGs) have red $H-K_{\mathrm{s}}>0.5$, at redshift

1 http://www.iso.vilspa.esa.es/ida/ 
$z \gtrsim 0.1$ the $K$-correction may shift normal galaxies to red $H-K_{\mathrm{s}}$ values ${ }^{2}$.

Active galactic nuclei (AGN) populate the colour range $K_{\mathrm{s}}-L W 2>2.7$ and, at redshift $z<1$, also $H-K_{\mathrm{s}}>0.5$ (Haas et al. 2004). While this range overlaps with that of the PAH-candidates, in this paper all sources with AGN signatures in their optical spectra are omitted. The results on the 33 ISO2MASS-AGN have been presented by Leipski et al. (2005, 2007, Papers 2+3).

The sample of $120 \mathrm{PAH}$-candidates is listed in Table 1. None of the 75 sources with optical spectra turned out to be a star. Therefore we adopt that the sample consists of 44 "red" galaxies with colours similar to those of AGN, and 76 "blue" galaxies outside the AGN colour range.

\section{Follow-up data}

\subsection{Optical spectroscopy}

In order to study the emission- and absorption-line properties of the PAH-candidates and to determine their redshifts, we have performed optical spectroscopy in the wavelength range between 3500 and $9000 \AA$. As mentioned above, among the red subset also AGN are expected. Therefore, we obtained spectra for the complete set of 44 red sources. For the remaining subset of 76 blue sources (120-44) we restricted the observational effort and obtained optical spectra of 19 sources randomly selected. Among these blue sources, no AGN was found.

The data were obtained during 2004 and 2005 at various telescopes $^{3}$, and standard data reduction was applied. The quality of the spectra is not homogeneous, but in general, it is sufficient to identify the prominent emission lines. Faint lines or absorption structures are measurable only in the best spectra of the brightest objects. Examples are shown in Fig. 2.

Good spectra of additional 12 sources are available from the Sloan Digital Sky Survey (SDSS, data release 7), as well as photometry of 43 sources for which we also inspected the morphology (Table 1).

\subsection{Mid-infrared spectroscopy}

In order to verify the PAH nature of the $6.7 \mu \mathrm{m}$ emission and to exclude the possibility of buried AGN, for the 19 brightest red sources we obtained low-resolution mid-infrared (MIR) spectra using the Infrared Spectrograph (IRS, Houck et al. 2004) onboard the Spitzer Space Telescope (Werner et al. 2004).

Spectra of 9 sources were observed in cycle-1 (prog-id 3231) covering the full wavelength range $5-38 \mu \mathrm{m}$ with a relatively short integration time $(4 \times 14 \mathrm{~s}$ in SL and $4 \times 30 \mathrm{~s}$ in LL). All 9 spectra show clear PAH features but poor $\mathrm{S} / \mathrm{N}$ in the high-excitation emission lines used for excluding or establishing buried AGN. Therefore, in cycle-2 (prog-id 20090) we observed 10 more sources with longer integration time $(12 \times 120 \mathrm{~s})$, but only in the range 19-38 $\mu$ m containing the high-excitation lines $[\mathrm{NeV}]_{24.3 \mu \mathrm{m}}$ and $[\mathrm{OIV}]_{25.9} \mu \mathrm{m}$. All 19 spectra were taken without peak-up images.

Starting from the background subtracted pipeline products, we performed standard interactive data reduction, using the

\footnotetext{
2 http://www.ipac.caltech.edu/2mass/releases/sampler/ sampler.html

3 South African Astrophysical Observatory $1.9 \mathrm{~m}$, Tautenburg 2m, Kitt Peak 2.1m, Calar Alto 2.2m, Nordic Optical Telescope $2.5 \mathrm{~m}$, Telescopio Nazionale Galileo 3.5 m, ESO/NTT 3.5m, CTIO Blanco 4m, ESO/VLT 8.2m.
}
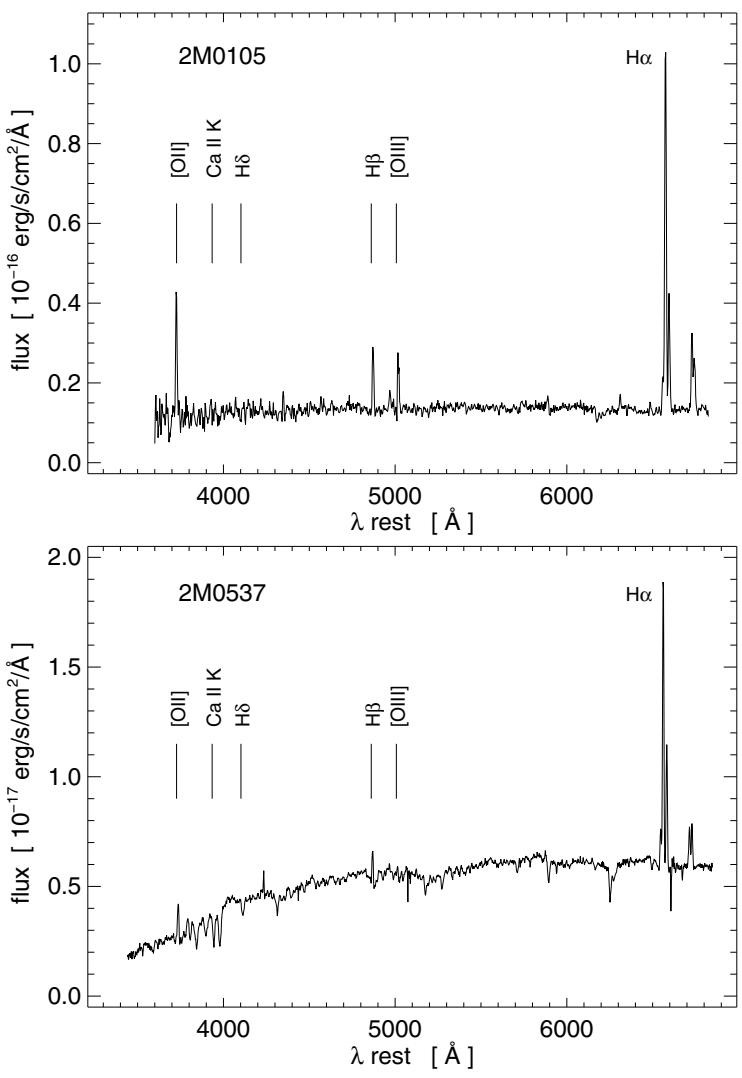

Fig. 2. Examples of optical spectra. Top (2MASS $01051501-2612466$ ): While $\mathrm{H}_{\alpha}$ and [O II] are prominent, $\mathrm{H}_{\beta}$ appears suppressed. Bottom (2MASS $05375494-4408547$ ): While $\mathrm{H}_{\alpha}$ is well seen, the other emission lines appear relatively faint and the $\mathrm{H}_{\delta}$ and Ca II K absorption is rather strong.

SPICE software tool as well as our own routines for improved cleaning of residual rogue pixels and and cosmic rays, and for combining the spectra of the four IRS channels.

Table 2 lists the MIR spectral properties as well as continuum fluxes at 15,24 and $35 \mu \mathrm{m}$.

Integration of the spectra over the $L W 2$ passband yields a good coincidence between the $L W 2$ and IRS photometry for all but one of the 9 sources $\left(F_{\mathrm{LW} 2} / F_{\mathrm{IRS}}=1.00 \pm 0.14\right)^{4}$.

\subsection{Mid- and far-infrared photometry}

All but 4 sources are not contained in the IRAS faint source catalogue. Therefore we analysed IRAS ADDSCANs ${ }^{5}$. Using a matching radius of $20^{\prime \prime}$ around the 2MASS position, 19 sources could be detected with at least $4 \sigma$ at $60 \mu \mathrm{m}$ and 13 of them also

4 The outlier (2MASS $15554606+1532218$ ) has a factor 1.8 lower IRS flux in the $L W 2$ passband. The discrepancy could be explained by either a photometric $L W 2$ error due to a non-detected cosmic ray event, or an IRS pointing error or aperture effects. On SDSS images the source is extended with a diameter of about $8^{\prime \prime}$ including a bright tail to the south. While from the $L W 2$ image the total flux is measured, part of the flux could be missed in the IRS spectrum due to the slit width of 3.7" between $5 \mu \mathrm{m}$ and $14 \mu \mathrm{m}$. However, the IRS spectrum of 2MASS 1555 does not show a jump at $14 \mu \mathrm{m}$ longward of which the slit width is $11.6^{\prime \prime}$. Probably a combination of all three effects is responsible for the discrepancy between $L W 2$ and IRS photometry of this source. Because this source has typical average properties, the results and conclusions are essentially not affected by the choice of the $6.7 \mu \mathrm{m}$ flux value.

5 http://scanpi.ipac.caltech.edu:9000/ 
at $100 \mu \mathrm{m}$. The fact that $13 / 19$ sources are detected in both filters supports that the detections are real. Because the flux level is rather low, we adopt in general a $30 \%$ photometric uncertainty, although for 4 sources listed in the IRAS faint source catalog our ADDSCAN-photometry agrees within $1 \%$. We also checked on the 2MASS and ISOCAM images that no nearby $\left(<30^{\prime \prime}\right)$ red source is likely to contribute to the IRAS fluxes. (Actually two more sources had been detected on ADDSCANs, but they have been discarded). Table 1 lists the photometry of the accepted detections and the $60 \mu \mathrm{m}$ upper limits of the remaining sources.

In the wavelength range 3.6-160 $\mu \mathrm{m}, 14$ sources are covered on archival maps taken with the Spitzer Space Telescope. All sources are detected in all bands observed and match well with the 2MASS positions (better than $2^{\prime \prime}$ for IRAC $3.6-8 \mu \mathrm{m}$ and $4-10^{\prime \prime}$ for MIPS $24-160 \mu \mathrm{m}$, respectively). Using the Post Basic Calibrated Data Products, we derived aperture photometry with an uncertainty of about $10 \%$ (IRAC) and less than $30 \%$ (MIPS). Because the used apertures are sufficiently large, e.g. $12^{\prime \prime}$ for IRAC, no aperture corrections are necessary. The values are listed in Table 3.

We computed far-infrared luminosities using the standard formula (Sanders \& Mirabel 1996, their Table 1):

$L_{\mathrm{FIR}}=4 \pi D_{L}^{2} \times 1.6 \times 1.26 \times 10^{-14}\left(2.58 F_{60}+F_{100}\right)\left[L_{\odot}\right]$, with $F_{60}$ and $F_{100}$ given in Jy. For the 6 cases with $60 \mu$ m detection but $100 \mu \mathrm{m}$ upper limit, we adopted $F_{100}=2 \times F_{60} . F_{100}$ is probably even higher, because most sources have $F_{60} / F_{100}>2$, if measured. Note that the $L_{\text {FIR }}$ formula assumes an average star forming galaxy SED and thus may weaken possible differences between M 82- and NGC 891-types. But given the photometric uncertainties, this approximation should be sufficient for our purpose, and any difference between the IRAS $60+100 \mu \mathrm{m}$ and Spitzer $70+160 \mu \mathrm{m}$ passbands should play a minor role. Therefore, we used the same formula also for the Spitzer 70 and $160 \mu \mathrm{m}$ data to derive $L_{\mathrm{FIR}}$, adopting $F_{60} \approx F_{70}$ and $F_{100} \approx F_{160}$ and $F_{160}=2 \times F_{70}$, when $F_{160}$ is not observed.

\subsection{K-correction}

Because our sources have strong PAH features as shown below, $K$-correction plays a role:

1) we have determined the $K$-correction for $L W 2$ fluxes from local ISO-SWS spectra of starforming galaxies. For the redshift of our sources from $z=0.03$ to $z=0.3$ the $K$-correction factor increases steadily from 1.1 to 2.2 ;

2) for $24 \mu \mathrm{m}$ fluxes we determined $K$-correction factors from our 19-38 $\mu \mathrm{m}$ spectra. To correct also the photometry data of sources without spectra, a fit yields F24 $(K$-corrected $)=(1+3 z) \times \mathrm{F} 24($ observed $)$

3 ) in the FIR $K$-correction plays a minor role, at least with regard to the measurement errors. At 60 and $70 \mu \mathrm{m}$ we applied a $K$-correction factor of $(1+0.33 z)$, i.e. 0 at $z=0$ and $10 \%$ at $z=0.3$, and no $K$-correction at 100 and $160 \mu \mathrm{m}$.

All data points shown in Figs. 8 to 10 have been $K$-corrected.

\section{Results and discussion}

\subsection{Optical spectra}

Two example spectra are displayed in Fig. 2. In general, the spectra show a range of blue and red continua as well as reddened Balmer emission lines with a ratio reaching $\mathrm{H}_{\alpha} / \mathrm{H}_{\beta}>6$ even after correction for stellar absorption troughs. Most spectra also show $[\mathrm{O} \mathrm{II}]_{3727} \AA$ emission (henceforth simply denoted [O II]).

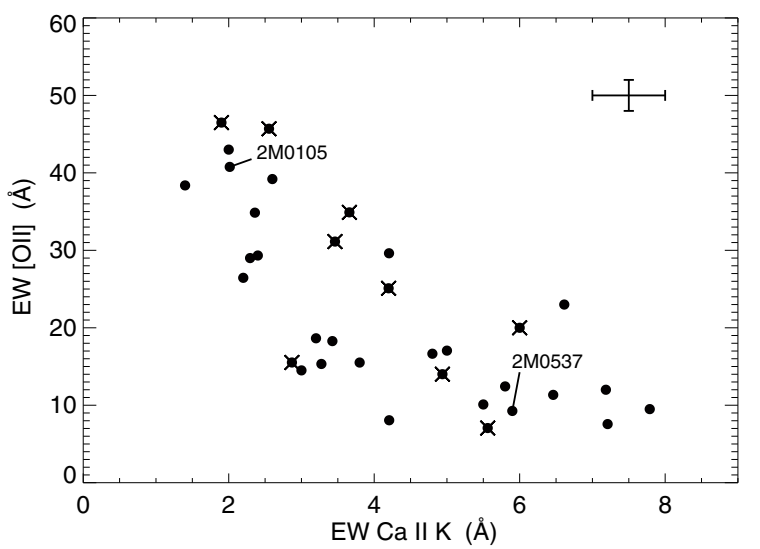

Fig. 3. Equivalent widths of [O II] emission and $\mathrm{Ca}$ II K absorption. The average errors are illustrated by the bars in the upper right corner. The two sources with spectra shown in Fig. 2 are labeled. Overplotted crosses mark those sources detected with IRAS at $60 \mu \mathrm{m}$. The two sources with the highest $E W[\mathrm{OII}]$ are the two ULIRGs of our sample.

The equivalent widths of [O II] and $\mathrm{H}_{\alpha}$ are broadly correlated. High-excitation emission lines ([O III $]_{5007} \AA$ ) mostly are not detected or are faint, and the emission line ratios place the objects in the starforming part of the standard diagnostic diagrams used for separating AGN, LINER and starforming galaxies (Veilleux \& Osterbrock 1987).

The equivalent width of [O II] is an indicator of the average massive star formation rate (e.g. Dressler \& Gunn 1982; Kennicutt 1992). Sodré \& Stasińska (1999) report a clear correlation of $E W[\mathrm{OII}]$ with spectral types ST obtained with a principal component analysis of the continuum and absorption features of spectra. For $S T>0$ their equivalent widths cover approximately the same range as in Fig. 3 of the present paper with $E W \sim 5 \AA$ for ST 0 and $E W \sim 40 \AA$ for ST $10^{6}$. These authors argue that the spectral sequence can be interpreted as a sequence of an increasing ratio of the present to the average past star formation rate. In that picture, low values of $E W[\mathrm{O} I I]$ correspond, on average, to galaxies where most of the star formation occured long ago, very few OB stars contribute to the optical spectrum, and the ionizing UV radiation field is dominated by AGB stars. The highest values of $E W[\mathrm{O} I \mathrm{II}]$ in our sample are measured for the two ULIRGS (Sect. 4.3).

From the $45 E W[\mathrm{O}$ II] measurements listed in Table 1, one finds a remarkably high mean value of $23 \AA$. This can be compared with the mean values of $11 \AA$ and $17 \AA$ found by Liu and Kennicutt (1995) for a complete, magnitude-limited sample of (optically selected) local galaxies and for a sample of more distant merger galaxies, respectively. Even for the (certainly unrealistic) case that the $\sim 30$ galaxies in our spectroscopic sample without $E W[\mathrm{OII}]$ measurements have no [O II] emission at all, the sample average of $14 \AA$ is larger than that of Liu \& Kennicutt's local sample.

About one third of the objects show $\mathrm{H}_{\delta}$ and/or Ca II K absorption with equivalent widths $E W>4 \AA$. This indicates that stars of intermediate age contribute strongly to the spectra. However, there is no galaxy in our spectroscopic sample with really strong Balmer absorption indicating a dominant A-star population as measured in "E+A" galaxies with $E W H_{\delta}>6 \AA$

6 The Sodré \& Stasińska (1999) spectral type ST is correlated with the Hubble morphological type. ST>0 roughly corresponds to Hubble types later than Sa, and ST 10 to irregular galaxies. 
(Liu \& Kennicutt 1995). The $\mathrm{H}_{\delta}$ and Ca II K absorptions suggest that about $0.5-1$ Gyr ago an intense starforming episode stopped and that we now see the galaxy in a post-starburst phase, but with considerable ongoing star formation as traced by the $\mathrm{H}_{\alpha}$ and [O II] emission.

The spectra of 34 sources had sufficient $\mathrm{S} / \mathrm{N}$ at the blue end to allow us to quantify both [O II] emission and Ca II K absorption. The equivalent widths of [O II] emission and $\mathrm{Ca}$ II $\mathrm{K}$ absorption are roughly anti-correlated. The sources with strong [O II] have weak Ca II K, and vice versa, as shown in Fig. 3.

So far, we have assumed that both the emission lines and their underlying continuum suffer, on average, from similar extinction. On the other hand, Calzetti (2001) found in a UVselected sample of star forming galaxies that the H II emission lines undergo a factor $\sim 2$ higher extinction than the stellar continuum. An explanation for this difference could be that H II regions are located closer to the parent dusty molecular clouds and therefore are more likely affected by extinction than the bulk of stars. If the difference between emission line and continuum extinction also holds for our sample, then one expects that $E W$ [O II] decreases with increasing $\mathrm{H}_{\alpha} / \mathrm{H}_{\beta}$ ratio (which traces the emission line extinction). In fact, the mean $E W[\mathrm{O}$ II] value decreases from $E W=26.6 \AA$ for sources with low $\mathrm{H}_{\alpha} / \mathrm{H}_{\beta}<6$ to $E W=14.2 \AA$ for high $\mathrm{H}_{\alpha} / \mathrm{H}_{\beta}>10$. However, the population in the lower right corner of Fig. 3 ( $E W[\mathrm{O}$ II] $<20$ and $E W$ $\mathrm{Ca}$ II $\mathrm{K}>4$ ) contains sources with low as well as high emission line extinction which again is not correlated with IRAS $60 \mu \mathrm{m}$ detection or non-detection. In addition, the highest $E W$ [O II] are seen in the two ULIRGs although they have $\mathrm{H}_{\alpha} / \mathrm{H}_{\beta} \geq 10$. This indicates that, even in the case of high emission line extinction, the [O II] equivalent width can identify the star forming activity fairly well and consistent with MIR emission line diagnostics (Sect. 4.2.2). Furthermore, the $E W$ [O II] comparison of our sample with that of others (Sodré \& Stasińska 1999; Kennicutt 1992; Liu \& Kennicutt 1995) was performed in a consistent manner, all samples not being extinction corrected. These considerations give us confidence that the potential extinction difference between emission lines and continuum does not affect our basic conclusions on the wide range of starforming activity and the presence of aged stellar populations in $\mathrm{PAH}$ selected galaxies.

We inspected the extent and morphology of 43 sources with SDSS images available. Most (34/43) of the sources appear extended $\left(>5^{\prime \prime}\right)$ and about one third (12/43) shows irregular morphology or possible faint companions (with unknown redshift). However the data did not allow us to establish a connection between morphology and spectral properties such as equivalent widths. Nevertheless, this suggests that disturbed morphology does not automatically imply the presence of powerful starbursts.

To summarise, the optical spectra indicate a range of ongoing starformation as well as evidence for a intermediate-age stellar populations. The basic conclusions have been drawn without calculating starforming rates from the emission line fluxes, because the corrections for extinction and for aperture loss due to the 1-2" slit widths introduce large uncertainties.

\subsection{Mid-infrared spectra}

\subsection{1. $\mathrm{PAH}$ nature of the $6.7 \mu \mathrm{m}$ flux}

While local non-AGN templates suggest that PAH emission dominates the $6.7 \mu \mathrm{m}$ flux of our sources, we need to exclude that hidden AGN contribute significantly to the $6.7 \mu \mathrm{m}$

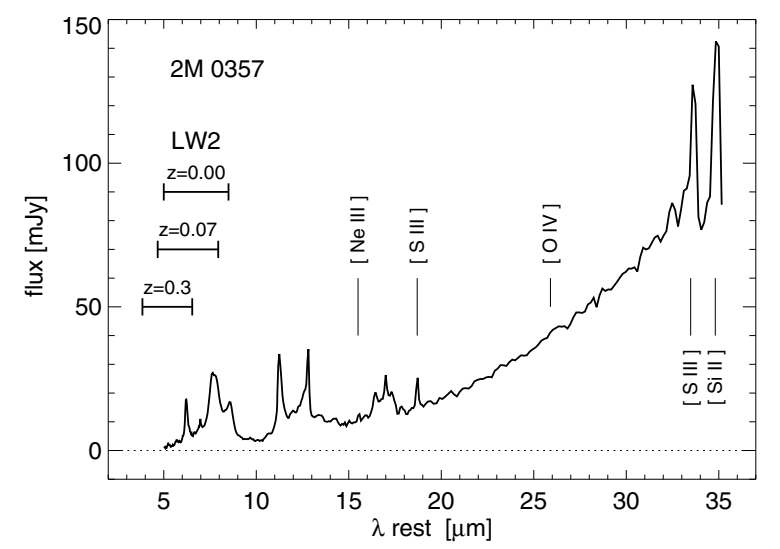

Fig. 4. Example IRS spectrum of 2MASS 03574895-1340458 exhibiting strong PAH features at $6-9 \mu \mathrm{m}$ and also at $11.3,12.7$ and $17 \mu \mathrm{m}$. Three emission lines are clearly detected: [S III] at 18.71 and $33.48 \mu \mathrm{m}$ and [Si II] at $34.81 \mu \mathrm{m}$, while [Ne III] at $15.55 \mu \mathrm{m}$ is very faint and [O IV] at $25.89 \mu \mathrm{m}$ is not detected. The horizontal bars indicate for three redshifts how much of the PAH features is covered by the $L W 2$ passband.

continuum. Hard X-ray data $(>2 \mathrm{keV})$ are not available for our sample. Therefore, we checked the nature of the $6.7 \mu \mathrm{m}$ flux by means of MIR spectra.

Figure 4 displays the IRS spectrum of the brightest source 2MASS 03574895-1340458. The 5-38 $\mu \mathrm{m}$ spectra of the other 8 cycle-1 sources look very similar. The MIR spectra show strong 6-9 $\mu \mathrm{m}$ PAH emission. Depending on the redshift of our sources $(0.03<z<0.3)$, the $L W 2$ passband essentially catches the $6.2 \mu \mathrm{m}$ PAH feature and part of the $7.7 \mu \mathrm{m}$ feature. The $8.6 \mu \mathrm{m}$ PAH feature is shifted out of $L W 2$.

On average, about $50 \%$ of the rest frame 6-9 $\mu \mathrm{m}$ PAH flux falls into the $L W 2$ passband and thus explains why the objects have high $K-L W 2>1.8$ colour values and meet the selection criterion. The individual values of the PAH fraction seen in $L W 2$ are listed in Table 2. Among our sample, however, we do not see a trend of $K-L W 2$ colour with redshift, indicating that the broad range of $K-L W 2$ (Fig. 1) is not dominated by $K$-correction effects.

Any $10 \mu \mathrm{m}$ silicate absorption is difficult to determine, but if present then it appears weak. With respect to buried AGN, which in principle could contribute to the $6.7 \mu \mathrm{m}$ continuum, we consider what can be concluded further from the 19-38 $\mu \mathrm{m}$ spectra of cycle-1 and cycle-2. High-excitation lines $\left([\mathrm{NeV}]_{24.3} \mu \mathrm{m}\right.$ and $\left.[\mathrm{OIV}]_{25.9} \mu \mathrm{m}\right)$ are not detected, even in the ten 19-38 $\mu \mathrm{m}$ spectra with longer integration times. The low upper limits of the line luminosities and equivalent widths argue against powerful buried AGN and against a significant $6.7 \mu \mathrm{m}$ AGN continuum. This conclusion is further supported when looking for possible reemission of shielding dust at longer optically thin wavelengths: With the exception of two ULIRGs, the SEDs show only a moderate rise from 15 to $35 \mu \mathrm{m}$, and hence are different from those of elusive X-ray detected AGN like NGC4945 and of starburstAGN composites like Circinus (Fig. 5). The two ULIRGs of our sample do not have a $5-10 \mu \mathrm{m}$ spectrum, but cool $F_{25} / F_{60}$ colours (Fig. 6) which argue in favour of starburst dominated $6.7 \mu \mathrm{m}$ emission with strong PAH features (Genzel et al. 1998).

These results from 19 sources with MIR spectra lead us to conclude that most if not all galaxies of our sample show strong PAH emission and that our LW2 photometry serves as a measure for about $50 \%$ of the 6-9 $\mu \mathrm{m}$ PAH luminosity. 


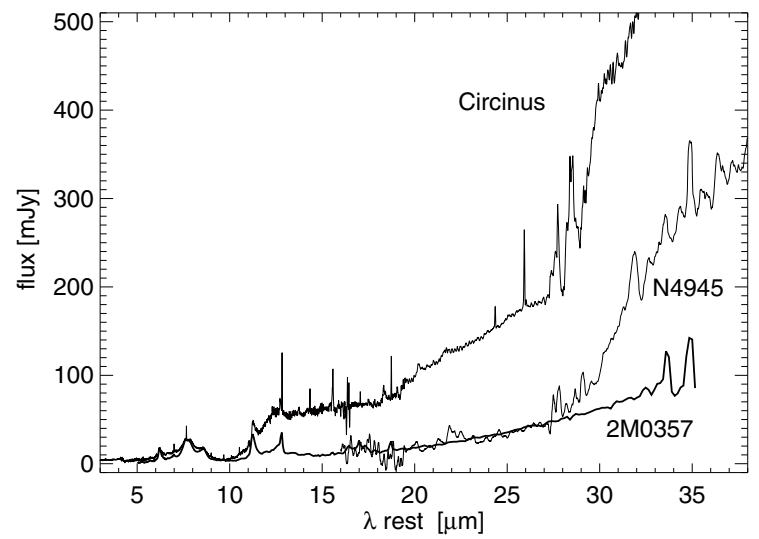

Fig. 5. IRS spectrum of 2MASS 03574895-1340458 and ISO-SWS spectra of the starburst-AGN composite Circinus and the elusive X-ray AGN NGC 4945. The ISO-SWS spectra are scaled to match the PAH flux level at 6-9 $\mu \mathrm{m}$. At wavelengths longer than $12 \mu \mathrm{m}$ and $30 \mu \mathrm{m}$, respectively, the continuum of Circinus and NGC 4945 is much brighter than that of $2 \mathrm{M} 0357$.

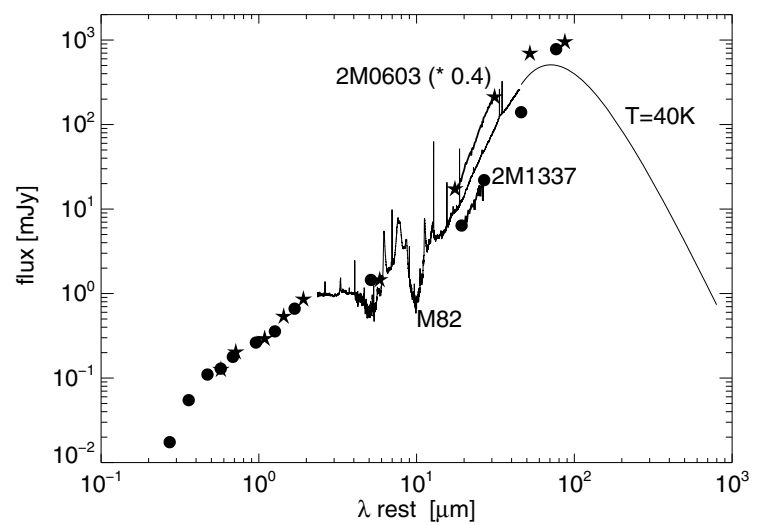

Fig. 6. SEDs of the two ULIRGs. 2MASS 06033357-4509412 (asterisks) is scaled by a factor 0.4 to match the $6.7 \mu \mathrm{m}$ flux of 2MASS $13371721+0904430$ (filled circles). In comparison with the spectrum of M 82, both SEDs show a similarly steep 15-30 $\mu \mathrm{m}$ rise. The temperature $T=40 \mathrm{~K}$ of the modified greybody is typical for ULIRGs when using an emissivity index $\beta=2$ (Klaas et al. 2001). The optical data are from SDSS.

\subsubsection{Mid-infrared starforming tracers}

$[\mathrm{Ne} \mathrm{II}]_{12.8} \mu \mathrm{m}$ would be the first choice among MIR starforming tracers, because of its brightness and the required low excitation potential of $21.6 \mathrm{eV}$. But in the low resolution spectra this line is not well separated from the $12.7 \mu \mathrm{m}$ PAH feature. Therefore we analysed the $[\mathrm{S} \mathrm{III}]_{18.7 \mu \mathrm{m}}$ and $[\mathrm{Ne} \mathrm{III}]_{15.5} \mu \mathrm{m}$ lines requiring excitations of $23.3 \mathrm{eV}$ and $40.9 \mathrm{eV}$, respectively. The fluxes are listed in Table 2. The [S III]/ [Ne III] flux ratio lies in the range 2-5 typical for (modestly) starforming galaxies; for comparison, intense starburst galaxies like M 82 have [S III]/ [Ne III] 2 (Verma et al. 2003) and the starburst knots in the overlapping region of the Antennae galaxy pair have [S III] / [Ne III] 1 (Kunze et al. 1996). This is because the radiation field of bursts of Ostars is harder and a higher fraction of neon is in the doubleionized state. There is a marginal trend that sources with high [Ne III] fraction also show a high equivalent width of $\mathrm{H}_{\alpha}$ and [O II] ${ }_{3727 \AA}$.

Two sources, which turn out to be ULIRGs (Sect. 4.3), have steeply rising 20-35 $\mu$ m continua (Fig. 6). But the MIR continua of all 17 other sources do not show the strong rise between 15

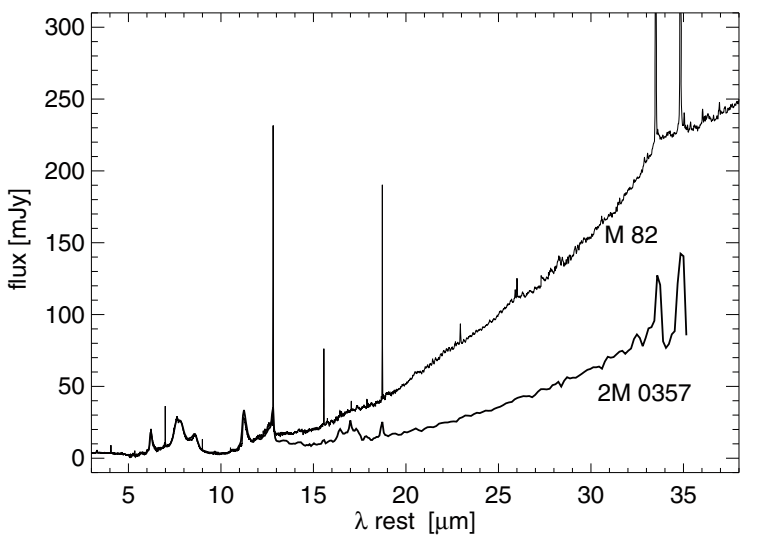

Fig. 7. IRS spectrum of 2MASS 03574895-1340458 and ISO-SWS spectrum of the starburst template M82. The spectrum of M82 is scaled to match the PAH flux level at 6-9 $\mu \mathrm{m}$. At wavelengths longer than $15 \mu \mathrm{m}$ the continuum of M82 is much brighter than that of $2 \mathrm{M} 0357$.

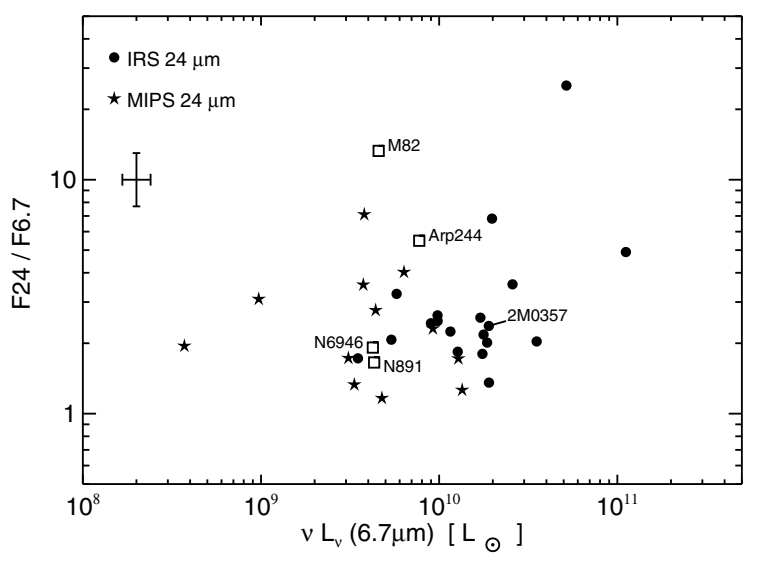

Fig. 8. Colour-magnitude diagram F24 $\mu \mathrm{m} / \mathrm{F} 6.7 \mu \mathrm{m}$ versus $6.7 \mu \mathrm{m}$ luminosity. The data have been $K$-corrected. Uncertainties of $20 \%$ in $x$ and $40 \%$ in $y$ are illustrated in the upper left corner. For comparison two spirals (NGC 891, NGC 6946), the Antennae Galaxy Arp 244 and the starburst galaxy M 82 are shown (open squares). Also labelled is 2MASS 0357, the source with MIR spectrum shown in Fig. 4.

and $35 \mu \mathrm{m}$ typical for warm starburst heated dust like that found in M 82 (Fig. 7).

This is also reflected in the low $F_{24} / F_{6.7}$ ratio for most sources as shown in Fig. 8. In this plot we included both the $F_{24}$ data from the spectra and the MIPS- $24 \mu$ m photometry (Tables 2 and 3); for three sources without redshift but with photometry we adopted the median redshift of the sample $(z=0.105)$. For the comparison sources we used the IRAS $25 \mu$ m fluxes from the NED (the difference between IRAS-25 and MIPS-24 bandpasses is negligible here). The $F_{24} / F_{6.7}$ colours of most galaxies appear more similar to those of the rather quiescent spiral galaxies NGC 891 (edge-on) and NGC 6946 (face-on) than to M 82. A few sources reach the $F_{24} / F_{6.7}$ ratio of Arp244, the colliding Antennae galaxy pair with dust-enshrouded starbursts as well as a large amount of cold dust (Mirabel et al. 1998; Haas et al. 2000).

To summarise, most ( 15/19) of the mid-IR spectra indicate rather modest starforming activity and do not show the signatures of powerful M 82-like starburst galaxies, either in emission lines or the continua. 

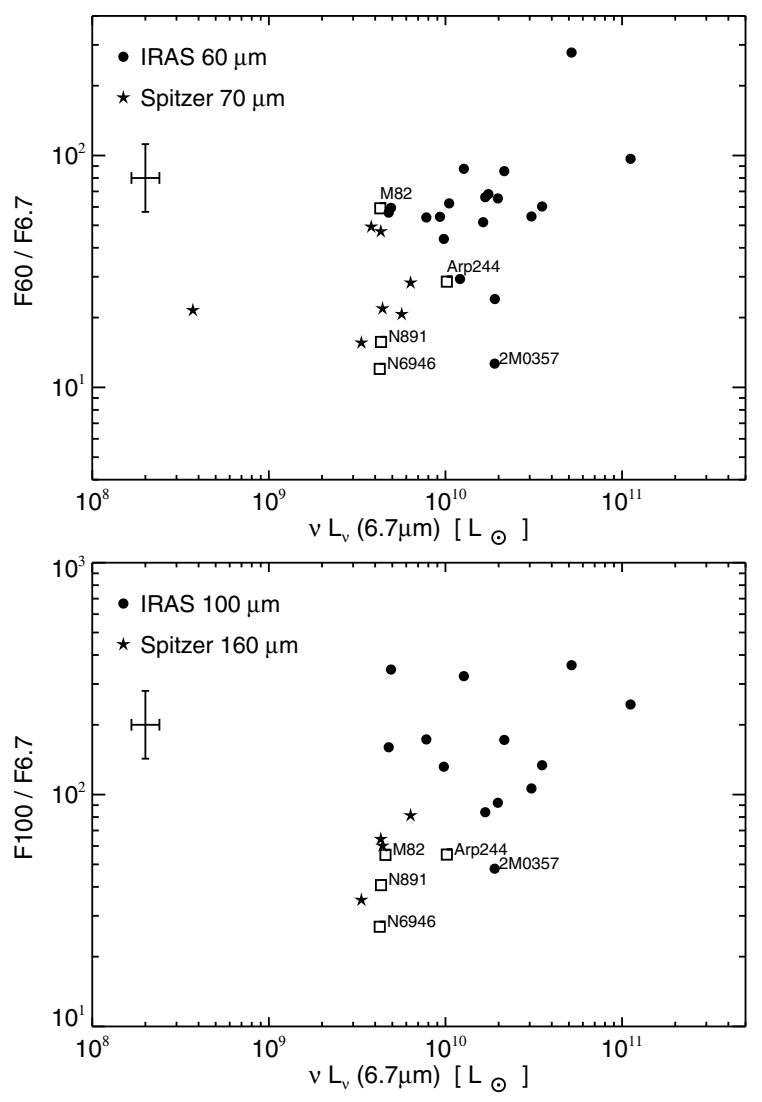

Fig. 9. Colour-magnitude diagrams F60/F6.7 and F100/F6.7 versus $6.7 \mu \mathrm{m}$ luminosity. The data have been $K$-corrected. Uncertainties of $20 \%$ in $x$ and $40 \%$ in $y$ are illustrated in the upper left corner. For comparison with local templates two spirals (NGC 891, NGC 6946), the Antennae Galaxy Arp 244 and the starburst galaxy M 82 are shown (open squares). Also labelled is 2MASS 0357, the source with the MIR spectrum shown in Fig. 4. The numerous $60 \mu \mathrm{m}$ upper limits populate the F60/F6.7 range between 10 and 100; they are not plotted to avoid confusion.

\subsection{Far-infrared properties}

Figure 9 shows the 60 and $100 \mu \mathrm{m}$ fluxes normalized by F6.7 $\mu \mathrm{m}$. We include also the Spitzer 70 and $160 \mu \mathrm{m}$ photometry, adopting for the first look that colour corrections play a minor role. A striking fact is that at least half of the FIR detected sources show a much higher $F_{60} / F_{6.7}$ ratio than the local cool templates, and the $F_{100} / F_{6.7}$ ratio of virtually all sources exceeds that of local templates. Thus the basic result is: Apart from the two ULIRGs, most if not all of the 17 sources with IRAS FIR detections are exceptionally strong cool dust emitters, relative to their PAH emission.

Further evidence for this conclusion comes from the farinfrared luminosities as shown in Fig. 10. Among the 19 IRAS detections 16 sources have $L_{\mathrm{FIR}}>10^{11} L_{\odot}$. Two of them qualify as ULIRGs with $L_{\text {FIR }}>10^{12} L_{\odot}$ (in Table 1 they are marked after the 2MASS name $)^{7}$. One of the two ULIRGs

\footnotetext{
7 We do not expect that our sample contains more than these two ULIRGs. The reason is that known local ULIRGs have $K_{\mathrm{s}}-L W 2>2.7$ and $H-K_{\mathrm{s}}>0.5$, and all objects of this red subsample have redshifts, so that the FIR luminosity can be determined. With exception of the two ULIRGs, all other sources of the red subsample have $L_{\mathrm{FIR}}<10^{12} L_{\odot}$ even in case of upper FIR flux limits. Outside of the red subsample a ULIRG could be expected if $z>0.3$. Then it still has $H-K_{\mathrm{s}}>0.5$, but $K_{\mathrm{s}}-L W 2$ becomes lower, because the PAH features move out of
}

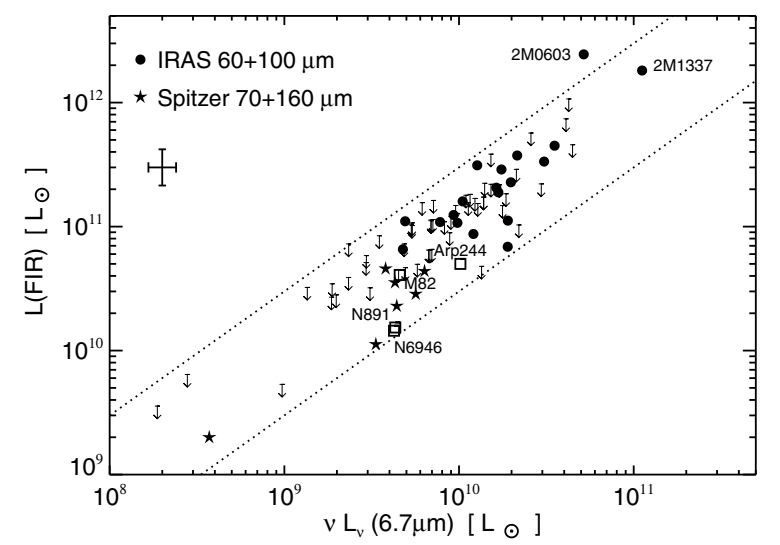

Fig. 10. Far-infrared versus $6.7 \mu \mathrm{m}$ luminosities. The data have been $K$-corrected. Upper limits from IRAS are marked with arrows. Uncertainties of $20 \%$ in $x$ and $40 \%$ in $y$ are illustrated in the upper left corner. For comparison with local templates, two spirals (NGC 891, NGC 6946), the Antennae Galaxy Arp 244 and the starburst galaxy $\mathrm{M} 82$ are shown (open squares). The dotted lines mark the range $L_{\mathrm{FIR}}=$ 3 and $30 \times L_{6.7}$. At highest luminosity, the $L_{\mathrm{FIR}} / L_{6.7}$ ratio tends to increase as indicated by the two labelled ULIRGs.

(2MASS13371721+0904430 at $z=0.3$ ) has a remarkably high F100/F60 (=5.5) indicating a large amount of cool dust despite the ultra-luminous IR emission. A similar cool ULIRG has been found at $z=0.45$ by Chapman et al. (2002) in the ISOFIRBACK survey.

Most of the sources with upper IRAS FIR flux limits have $L_{\text {FIR }}\left(<10^{11} L_{\odot}\right)$. The seven Spitzer FIR detections reach about a factor of 5 lower fluxes than the IRAS upper limits, resulting in both lower $L_{\mathrm{FIR}}$ and $L_{\mathrm{FIR}} / L_{6.7}$, too, which makes the sources similar to local cool templates. Therefore, we suggest that most of the sources with IRAS FIR upper limits also have $L_{\text {FIR }} / L_{6.7}$ similar to the Spitzer FIR detected sources and to local cool templates.

From Fig. 10 it is clear that $L_{\mathrm{FIR}}$ is basically correlated with $L_{6.7}$. However, the $L_{\mathrm{FIR}} / L_{6.7}$ ratio of the entire sample varies between 3 and 30, in a range of a factor of 10 . The ratio increases further at highest luminosities, consistent with the results by Klaas et al. (2001) and Elbaz et al. (2002). If the total IR luminosity $L_{3-1000} \mu \mathrm{m}$ is considered instead of $L_{\mathrm{FIR}}$, the range of $L_{3-1000 \mu \mathrm{m}} / L_{6.7}$ will be even larger.

Comparing the FIR properties with optical equivalent widths, the IRAS detected sources have the same $E W[\mathrm{O} I \mathrm{II}$ versus $E W$ Ca II K distribution as the IRAS non-detections and do not show a preference for a high $E W[\mathrm{O}$ II] (Fig. 3). In particular, for those sources with low $E W[\mathrm{O} I \mathrm{II}$, the cool dust temperature and the signatures of old stellar populations suggests that the FIR luminosity is powered substantially by the interstellar radiation field from the old stars rather than purely by the ongoing starformation. If this is the case, then $L_{\mathrm{FIR}}$ will not properly measure the ongoing starforming activity.

To summarise, most (17/19) of the IRAS $60-100 \mu \mathrm{m}$ detected sources are exceptionally luminous cool dust emitters, but not all of them exhibit strong starforming signatures as traced by, for instance, their [O II] equivalent widths.

the $L W 2$ passband. In this colour range there are 10 sources without spectra, but for none of them is the optical to MIR SED consistent with a ULIRG template at (photometric) redshift $z>0.2$. 


\subsection{Optical to millimetre SEDs}

Figure 11 shows the mean optical to millimetre SEDs $\left(v \cdot F_{v}\right)$ of the 17 IRAS sources and of the 7 Spitzer sources, detected at FIR wavelengths. The two ULIRGs have been excluded. The important features of these IRAS- and Spitzer-SEDs are:

1) The Spitzer-SED has three peaks of roughly the same height, one in the optical at about $0.6 \mu \mathrm{m}$, one in the MIR around $7 \mu \mathrm{m}$ and one in the FIR at about $100 \mu \mathrm{m}$. Between these peaks the SED shows valleys at about 4 and $25 \mu \mathrm{m}$. This SED is similar to that of NGC 891 and NGC 6946.

2) The IRAS-SED shows peaks of about the same height at optical and MIR wavelengths, but is about 7-10 times brighter in the FIR than the Spitzer-SED. At $6.7 \mu \mathrm{m}$ the IRAS-SED is about a factor of 1.5 higher than the Spitzer-SED. In the optical-NIR the IRAS-SED is somewhat redder than the Spitzer-SED. ${ }^{8}$ The redder optical SED is consistent with the IRAS detection statistics: While among the 44 sources with red NIR SED (i.e., with $H-K>0.5$ in Fig. 1) about one quarter is detected with IRAS, the fraction of the IRAS detections among the remaining 76 bluer sources is about a factor of 3 lower. Thus, optical selection criteria are biased against the red but exceptionally FIR bright sources.

3) Figure 11 also shows the mean $850 \mu$ m flux predicted for the IRAS sources. This prediction is based on the $1: 1$ relation between $L W 2$ and SCUBA $850 \mu$ m flux found for normal, luminous and ultra-luminous dust-rich local galaxies (Klaas et al. 2001; Haas et al. 2002). The remarkable benefit of this relation is that the sub-mm flux can be predicted from the PAH flux, despite the fact that the emission from cold dust is not related to that from transiently heated very small grains. The $60-850 \mu \mathrm{m} \mathrm{SED}$ is consistent with cool dust at $T \lesssim 25 \mathrm{~K}$ (emissivity index $\beta=2$ ). Here, we applied a $K$-correction for average flux loss in $L W 2$. Without such a $K$-correction the dust temperature would be warmer. Using standard formulae (Krügel 2003), high dust and gas masses are derived $\left(M_{\text {dust }}=10^{8}-10^{9} M_{\odot}\right.$, and $M_{\text {gas }}=10^{10}-10^{11} M_{\odot}$ assuming the standard gas/dust mass ratio of 100). For comparison, the dust masses of M 31 (Andromeda galaxy) and the archetypal ULIRG Arp 220 are about $3 \times 10^{7}$ and $1.4 \times 10^{9} M_{\odot}$, respectively (Haas et al. 1998; Klaas et al. 2001).

4) For comparison, the Milky Way SED from COBE/DIRBE is shown. While the peaks at 6.7 and $100 \mu \mathrm{m}$ are of similar height, the MIR 20-60 $\mu \mathrm{m}$ energy output is much lower than in the IRAS- and Spitzer-SEDs, and at optical wavelengths the Milky Way would be about ten times brighter than in the FIR (not shown in Fig. 11 to avoid confusion by too many lines). The SEDs of sources with an IRAS FIR upper limit have about equally high peaks at optical and $6.7 \mu \mathrm{m}$. This adds a further argument supporting that these sources are similar to the Spitzer-SED sources, i.e. local templates like NGC 891 and NGC 6946.

To summarise, the most striking feature of the optical to millimetre SEDs is the strong FIR bump in about $15 \%(17 / 120)$ of the sources. Despite being caused by rather cold dust, the FIR energy output of these sources is about a factor of ten higher than at optical wavelengths. Future surveys, for instance with the Herschel Space Telescope, will find more such galaxies, also at higher redshifts, and thus may solve the debate on how much a

\footnotetext{
${ }^{8}$ Because the number of our Spitzer FIR detected sources covered by SDSS is very small, we used the mean SDSS SED of all non-IRAS detections. The result is basically the same, but smoother.
}

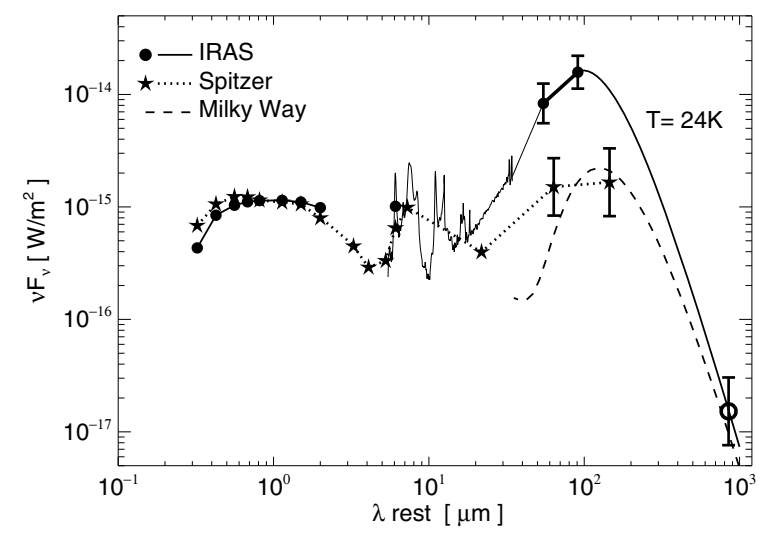

Fig. 11. Mean optical to millimetre SEDs $\left(v \cdot F_{v}\right)$ of two subsamples. The upper FIR data points (filled circles) are the IRAS detections; the two ULIRGs have been excluded. The lower FIR photometry curve (asterisks) refers to the Spitzer FIR detections. The mean F6.7 of the IRAS sample is a factor of 1.5 higher than for the Spitzer sample. The IRS spectrum is that of 2MASS 0357 (Fig. 4), scaled to the mean F6.7 of the IRAS sample. The optical and NIR data are from SDSS and 2MASS. The modified greybody with temperature $T=24 \mathrm{~K}$ has an emissivity index $\beta=2$. The open circle marks the predicted $850 \mu \mathrm{m}$ flux adopting the F6.7/F850 relation (Haas et al. 2002). The long-dashed line shows Milky Way cirrus of the solar neighbourhood measured with COBE/DIRBE; it is scaled to the mean Spitzer $6.7 \mu \mathrm{m}$ flux level.

cool galaxy population contributes to the cosmic infrared background (e.g. Lagache et al. 2005; Dole et al. 2006; Juvela et al. 2009).

\section{Summary and conclusions}

In order to obtain a census of the typical properties of PAH emitting galaxies, we combined the ISOCAM Parallel Survey at $6.7 \mu \mathrm{m}$ with $2 \mathrm{MASS}$ and colour-selected a sample of 120 candidates for strong PAH emission. Optical spectra of 75 sources establish that they are starforming galaxies at moderate distance $(0.03<z<0.3)$ with a median redshift $z \sim 0.1$. Midinfrared spectroscopy of the reddest 19 sources confirms that they have strong PAH emission and that they are not dustenshrouded AGN. This leads us to conclude that the entire sample of 120 sources consists of PAH selected galaxies with a $6.7 \mu$ m luminosity between $10^{8}$ and $10^{11} L_{\odot}$. This galaxy population has the following properties:

1) In about one third of the sources the $\mathrm{H}_{\alpha}$ and $[\mathrm{O} \mathrm{II}]_{3727 \AA}$ equivalent widths indicate intermediate to strong starforming activity, while one third shows only moderate ongoing star formation but $\mathrm{H}_{\delta}$ and/or Ca II K absorption, indicating a significant contribution of stars of intermediate mass and age. The remaining third of the sources lies between these cases. Many sources exhibit morphological irregularities, but our data did not allow us to establish a relation with spectral properties.

2) The mid-infrared emission lines $[\mathrm{S} \mathrm{III}]_{18.7 \mu \mathrm{m}}$ and $[\mathrm{Ne} \mathrm{IIII}]_{15.5 \mu \mathrm{m}}$ as well as the lack of higher ionisation lines corroborate that even in the reddest sources the radiation field is relatively soft. For all sources except two ULIRGs, the MIR lines and continua exclude powerful buried AGN and do not suggest the presence of intense hidden starbursts. The $24 \mu \mathrm{m} / 6.7 \mu \mathrm{m}$ flux ratio is more like that of cool normal spiral galaxies such as NGC 891 
and NGC 6946 and in a few sources it reaches that of the Antennae galaxy Arp 244.

$3)$ In the far-infrared, 26 sources are detected on IRASADDSCANS or Spitzer maps. Most SEDs steeply rise from 60 to $100 \mu \mathrm{m}$, adding further evidence that a large amount of cool dust $\left(T<25 K, M_{\text {dust }}>10^{8} M_{\odot}\right)$ dominates the FIR luminosity $\left(10^{9}\right.$ to $\left.2 \times 10^{12} L_{\odot}\right)$. In 17 sources $(\sim 15 \%)$ the FIR energy output is 5-10 times higher than the optical one, despite the cool dust temperature. The sources with upper FIR flux limits are consistent with this picture. The exceptional FIR-luminous galaxies increase the dispersion in the $L_{\mathrm{PAH}} / L_{\mathrm{FIR}}$ relation. Their, on average, redder optical colours and the fact that $30 \%$ of them have rather low $\mathrm{H}_{\alpha}$ and $[\mathrm{O} \mathrm{II}]_{3727 \AA}$ equivalent widths, strongly suggest that optical selection criteria fail to collect a complete PAH emitting galaxy sample.

At moderate distance, PAH selected galaxies turn out to be a quite heterogeneous population of dust-rich, partly infraredluminous galaxies but mostly cool with a range of post-starburst signatures and starforming activity which appears rather modest relative to the entire gas content. The diversity of PAH emitting galaxies strongly suggests that the cosmological interpretation of deep 15 and $24 \mu \mathrm{m}$ surveys in favour of an early universe full of IR starburst galaxies with high starforming efficiency appears to be premature.

Acknowledgements. The ISOCAM Parallel Survey has been performed with the Infrared Space Observatory ISO, an ESA project with instruments funded by ESA Member States and with the participation of ISAS and NASA. The Two Micron All Sky Survey (2MASS) is a joint project of the University of Massachusetts and IPAC/Caltech, funded by the National Aeronautics and Space Administration and the National Science Foundation. This work is based essentially on observations made with the Spitzer Space Telescope, which is operated by the Jet Propulsion Laboratory, California Institute of Technology under a contract with NASA. Observing time for optical spectroscopy has been granted at the telescopes: South African Astrophysical Observatory $1.9 \mathrm{~m}$, Tautenburg $2 \mathrm{~m}$, Kitt Peak 2.1m, Calar Alto 2.2m, Nordic Optical Telescope 2.5m, Telescopio Nazionale Galileo 3.5m, ESO/NTT 3.5m, CTIO Blanco 4m, ESO/VLT 8.2m. We thank an anonymous referee for detailed constructive comments. This work was supported by Nordrhein-Westfälische Akademie der Wissenschaften und der Künste.

\section{References}

Bendo, G. J., Draine, B. T., Engelbracht, C. W., et al. 2008, MNRAS, 389, 629 Bregman, J. D., Bregman, J. N., \& Temi, P. 2008, ASPC, 381, 34

Calzetti, D. 2001, PASP, 113, 1449

Caputi, K. I., Lagache, G., Yan, L., et al. 2007, ApJ, 660, 97
Cesarsky, C. J., Abergel, A., Agnese, P., et al. 1996a, A\&A, 315, L32 Cesarsky, D., Lequeux, J., Abergel, A., et al. 1996b, A\&A, 315, L309 Chapman, S. C., Smail, I., Ivison, R. J., et al. 2002, ApJ, 573, 66 Chini, R., Kreysa, E., Kruegel, E., et al. 1986, A\&A, 166, L8 Dale, D. A., Helou, G., Contursi, A., et al. 2001, ApJ, 549, 215 Dale, D. A., Bendo, G. J., Engelbracht, C. W., et al. 2005, ApJ, 633, 857 Dennefeld, M., Lagache, G., Mei, S., et al. 2005, A\&A, 440, 5 Dole, H., Lagache, G., Puget, J.-L., et al. 2006, A\&A, 451, 417 Dressler, A., \& Gunn, J. E. 1982, ApJ, 263, 533

Elbaz, D., Cesarsky, C. J., Chanial, P., et al. 2002, A\&A, 384, 848 Genzel, R., Lutz, D., Sturm, E., et al. 1998, ApJ, 498, 579

Haas, M., Lemke, D., Stickel, M., et al. 1998, A\&A, 338, L33 Haas, M., Klaas, U., Coulson, I., et al. 2000, A\&A, 356, L83 Haas, M., Klaas, U., \& Bianchi, S. 2002, A\&A, 325, L23

Haas, M., Siebenmorgen, R., Leipski, C., et al. 2004, A\&A, 419, L49 Houck, J. R., Roellig, T. L., van Cleve, J., et al. 2004, ApJS, 154, 18 Juvela, M., Mattila, K., Lemke, D., et al. 2009, A\&A, 500, 763 Kaneda, H., Onaka, T., Sakon, I., et al. 2008, ApJ, 684, 270 Kennicutt, R. C. 1992, ApJ, 388, 310

Klaas, U., Haas, M., Müller, S. A. H., et al. 2001, A\&A, 379, 823

Krügel, E. 2003, The physics of interstellar dust, IoP Series in Astron. \& Astrophys. Bristol

Kunze, D., Rigopoulou, D., Lutz, D., et al. 1996, A\&A, 315, L101

Lagache, G., Puget, J.-L., \& Dole, H. 2005, ARA\&A, 43, 727

Laurent, O., Mirabel, I. F., Charmandaris, V., et al. 2000, A\&A, 359, 887

Leipski, C., Haas, M., Meusinger, H., et al. 2005, A\&A, 440, L8

Leipski, C., Haas, M., Meusinger, H., et al. 2007, A\&A, 464, 895

Lemke, D., Mattila, K., Lehtinen, K., et al. A\&A, 331, 742

Liu, C. T., \& Kennicutt, R. C. 1995, ApJ, 450, 547

Mattila, K., Lemke, D., Haikala, L. K., et al. 1996, A\&A, 315, L353

Mattila, K., Lehtinen, K., Lemke, D. 1999, A\&A, 342, 643

Mirabel, I. F., Vigroux, L., Charmandaris, V., et al. 1998, A\&A, 333, L1

Monkiewicz, J. A., Dickinson, M. E., Davoodi, P., et al. 2008, ASPC, 381, 332

Ott, S., Siebenmorgen, R., Schartel, N., et al. 2003, ESA SP-511, 159

Patris, J., Dennefeld, M., Lagache, G., et al. 2003, A\&A, 412, 349

Puget, J. L., \& Léger, A. 1989, ARA\&A, 27, 161

Rowan-Robinson, M., Lari, C., Perez-Fournon, I., et al. 2004, MNRAS, 315, 1290

Sajina, A., Scott, D., Dennefeld, M., et al. 2006, MNRAS, 369, 939

Sanders, D. B., \& Mirabel, I. F. 1996, ARA\&A, 34, 749

Schweitzer, M., Lutz, D., Sturm, E., et al. 2006, ApJ, 649, 79

Shi, Y., Ogle, P., Rieke, G. H., et al. 2007, ApJ, 669, 841

Siebenmorgen, R., Abergel, A., Altieri, B., et al. 1996, A\&A, 315, L169

Siebenmorgen, R., Schartel, N., \& Ott, S. 2000, LNP 548, 275 in ISO Surveys of a Dusty Universe, ed. D. Lemke et al., 275

Sodré, L., \& Stasińska, G. 1999, A\&A, 345, 391

Thronson, H. A., Jr., Walker, C. K., et al. 1987, ApJ, 318, 645

Uchida, K. I., Sellgren, K., Werner, M. W., et al. 2000, ApJ, 530, 817

Väisänen, P., Morel, T., Rowan-Robinson, M., et al. 2002, MNRAS, 337, 1043

Veilleux, S., \& Osterbrock, D. E. 1987, ApJS, 63, 295

Verma, A., Lutz, D., Sturm, E., et al. 2003, A\&A, 403, 829

Werner, M. W., Roellig, T. L., Low, F. J., et al. 2004, ApJS, 154, 1

Wu, H., Cao, C., Hao, C.-Na, et al. 2005, ApJ, 632, L79

Xu, C. 2000 ApJ, 541, 134

Zhu, Yi-N., Wu, H., Cao, C., et al. 2008, ApJ, 686, 155 
M. Haas et al.: Polycyclic aromatic hydrocarbon selected galaxies, Online Material $p$ I

Table 1. The sample of PAH selected galaxies and observed properties.

\begin{tabular}{|c|c|c|c|c|c|c|c|c|c|c|c|c|c|c|}
\hline 2MASS & $\begin{array}{c}L W 2 \\
\mathrm{mJy} \text { a) }\end{array}$ & $\begin{array}{c}H \\
\mathrm{mag}\end{array}$ & $\begin{array}{c}K_{s} \\
\mathrm{mag}\end{array}$ & $\begin{array}{r}F_{60} \\
\mathrm{mJy} \mathrm{f})\end{array}$ & $\begin{array}{l}F_{100} \\
\text { mJy }\end{array}$ & $\overline{z z}$ & $\begin{array}{r}\mathrm{H} \alpha \\
\mathrm{b})\end{array}$ & $\begin{array}{r}\mathrm{H} \beta \\
\mathrm{b})\end{array}$ & $\begin{array}{r}{[\mathrm{OII}]} \\
\mathrm{b})\end{array}$ & $\begin{array}{r}E W \mathrm{H} \alpha \\
\AA \mathrm{c})\end{array}$ & $\begin{array}{r}E W[\mathrm{OIII}] \\
\AA \mathrm{c})\end{array}$ & $\begin{array}{r}E W \mathrm{H} \delta \\
\AA \mathrm{d})\end{array}$ & $\begin{array}{r}\text { EW Ca II K } \\
\AA \text { d) } \\
\end{array}$ & $\begin{array}{l}\text { Morph } \\
\text { e) }\end{array}$ \\
\hline $00112015-1156099$ & 0.98 & 15.81 & 15.47 & $<144$ & & 0.241 & 2.33 & 0.78 & & -53.19 & & 3.75 & & \\
\hline 00180953-1009448 & 0.77 & 16.01 & 15.10 & $<129$ & & & & & & & & & & compact \\
\hline $00253965-3252466$ & 2.58 & 15.41 & 15.19 & $<147$ & & & & & & & & & & \\
\hline $00285431-7726146$ & 3.45 & 15.28 & 14.78 & 190 & 590 & 0.087 & 10.76 & 0.91 & 2.11 & -46.98 & -31.13 & 5.22 & 3.46 & \\
\hline $00445167-2653191$ & 1.73 & 15.51 & 14.73 & $<132$ & & 0.113 & 9.52 & 0.70 & 2.06 & -18.42 & -8.06 & 5.48 & 4.21 & \\
\hline $00465933-2527218$ & 1.37 & 15.71 & 15.50 & $<123$ & & & & & & & & & & \\
\hline $00482481-2519270$ & 0.95 & 15.34 & 15.41 & $<138$ & & & & & & & & & & \\
\hline $00524246-3024175$ & 1.51 & 15.40 & 14.90 & $<129$ & & & & & & & & & & \\
\hline $00544355+0024382$ & 1.99 & 15.88 & 15.83 & $<132$ & & & & & & & & & & edge-on \\
\hline $00544465-3907006$ & 3.12 & 15.90 & 15.44 & $<135$ & & & & & & & & & & \\
\hline $00561930-3742185$ & 1.08 & 16.10 & 15.21 & $<96$ & & 0.090 & 2.20 & & & -7.50 & & 3.80 & & \\
\hline $00565066-2736010$ & 1.05 & 16.36 & 15.78 & $<114$ & & 0.109 & 5.80 & 0.72 & 1.44 & -45.90 & -14.10 & 2.20 & & \\
\hline $01051501-2612466$ & 1.71 & 16.15 & 15.43 & $<132$ & & 0.110 & 11.97 & 2.08 & 4.14 & -94.84 & -40.77 & 2.86 & 2.01 & \\
\hline 01144234-4521493 & 0.96 & 16.17 & 15.38 & $<96$ & & 0.123 & 9.31 & 0.85 & & -54.94 & & & & \\
\hline $01240481+0403022$ & 1.57 & 15.90 & 15.33 & $<120$ & & 0.110 & 9.00 & 0.93 & & -42.00 & & 2.60 & & \\
\hline $01255879+8739093$ & 2.13 & 15.93 & 15.55 & $<162$ & & & & & & & & & & \\
\hline 01511176-0931553 & 0.80 & 15.86 & 14.92 & $<150$ & & & & & & & & & & compact \\
\hline $01515168+0253146$ & 1.40 & 15.67 & 15.11 & $<123$ & & 0.128 & 8.67 & 0.75 & 1.40 & -32.19 & -9.50 & 1.50 & 7.79 & \\
\hline $02072273+2338418$ & 2.04 & 13.74 & 13.62 & $<81$ & & & & & & & & & & \\
\hline $02185851-0242460$ & 1.53 & 15.51 & 15.26 & $<156$ & & & & & & & & & & \\
\hline $02261823-1510595$ & 1.40 & 15.56 & 15.19 & $<111$ & & 0.070 & 38.43 & 5.01 & & -35.91 & & 2.00 & & \\
\hline $02542961+1509122$ & 1.36 & 16.14 & 14.92 & $<147$ & & 0.099 & 25.43 & 1.31 & & -40.30 & & 6.00 & 6.61 & \\
\hline $03262563+3039153$ & 2.97 & 14.73 & 14.60 & $<138$ & & & & & & & & & & \\
\hline $03280979+3126028$ & 1.54 & 14.23 & 13.90 & $<210$ & & & & & & & & & & \\
\hline $03525104+1120254$ & 1.04 & 15.39 & 14.77 & $<156$ & & & & & & & & & & compact \\
\hline $03561911-6423202$ & 1.03 & 15.82 & 15.33 & & & 0.059 & 34.12 & 9.24 & & -78.06 & & 2.00 & & \\
\hline $03574895-1340458$ & 10.67 & 14.52 & 13.87 & 160 & 620 & 0.072 & 42.70 & 3.76 & & -54.02 & & 3.00 & 2.40 & \\
\hline $04145870+0547135$ & 1.91 & 15.49 & 15.09 & $<96$ & & 0.096 & 4.44 & 0.84 & 1.38 & -85.28 & -49.82 & 2.00 & & \\
\hline 05375494-4408547 & 1.47 & 15.48 & 15.04 & $<78$ & & 0.105 & 1.41 & 0.14 & 0.22 & -24.12 & -9.27 & 2.96 & 5.87 & \\
\hline $05400261-6002387$ & 2.26 & 15.38 & 14.39 & 210 & 430 & 0.155 & 12.00 & & 3.61 & -30.00 & -20.00 & 4.30 & 6.00 & \\
\hline $05575775-3808188$ & 1.15 & 15.15 & 14.81 & $<60$ & & 0.102 & 14.00 & & & -34.00 & & & & \\
\hline $05580218-3805258$ & 0.88 & 15.57 & 14.91 & $<87$ & & 0.033 & 9.50 & & & -12.80 & & & & \\
\hline 06033357-4509412 u & 3.51 & 15.28 & 14.19 & 1680 & 2300 & 0.161 & 16.00 & -1.14 & 4.46 & -48.00 & -46.49 & 5.48 & 1.90 & \\
\hline $06213825+7813105$ & 1.76 & 14.71 & 14.19 & $<99$ & & & & & & & & & & \\
\hline $08083959+7554555$ & 0.76 & 15.51 & 14.89 & $<114$ & & & & & & & & & & \\
\hline $11110523+5550119$ & 1.17 & 15.82 & 14.98 & $<108$ & & 0.141 & 11.01 & 2.10 & 2.35 & -59.09 & -18.28 & 2.10 & 3.42 & \\
\hline $12191852+2953166$ & 3.06 & 14.99 & 14.46 & $<213$ & & 0.104 & 10.09 & & 1.70 & -16.11 & -9.30 & & & tail \\
\hline $12211572+1151156$ & 0.99 & 15.58 & 15.18 & $<117$ & & & & & & & & & & group \\
\hline $12255713+4838051$ & 0.83 & 16.31 & 15.49 & $<174$ & & 0.261 & 7.86 & 1.84 & & -41.30 & & & & irregular \\
\hline $12281094+0852471$ & 2.29 & 15.43 & 15.07 & $<114$ & & 0.088 & 53.90 & 12.40 & 28.00 & -50.77 & -39.20 & 1.80 & 2.60 & regular \\
\hline $12325569+1328585$ & 1.93 & 16.23 & 15.99 & $<111$ & & & & & & & & & & companion? \\
\hline $12361826-3938464$ & 1.53 & 15.84 & 15.10 & $<138$ & & 0.105 & 8.17 & 1.99 & & -27.74 & & & & \\
\hline $12361865-3938006$ & 3.19 & 15.56 & 15.10 & 230 & & 0.109 & 1.64 & 0.17 & 0.23 & -43.24 & -29.62 & 2.19 & 4.20 & \\
\hline $12482307-0344102$ & 1.87 & 15.26 & 14.55 & 200 & 470 & 0.176 & 10.98 & 0.72 & 2.55 & -23.80 & -14.01 & 5.80 & 4.94 & regular \\
\hline $13092436+2939351$ & 1.26 & 16.23 & 15.51 & $<168$ & & 0.113 & 9.64 & 0.80 & 7.70 & -33.56 & -15.00 & & & companion? \\
\hline $13165999-3336459$ & 3.53 & 15.00 & 14.29 & 120 & & 0.111 & 4.19 & 0.15 & 0.42 & -13.75 & -7.06 & 3.70 & 5.56 & \\
\hline $13371372+0906127$ & 1.41 & 15.84 & 15.33 & $<150$ & & 0.138 & 26.96 & 4.35 & 13.01 & -35.34 & -29.00 & 2.10 & 2.29 & edge-on \\
\hline $13371721+0904430$ u & 1.45 & 16.10 & 14.93 & 140 & 780 & 0.306 & 17.03 & 1.89 & 7.20 & -52.67 & -45.70 & 4.50 & 2.55 & companion \\
\hline $13371806-3005123$ & 0.92 & 15.99 & 15.59 & $<156$ & & 0.037 & 20.90 & 6.20 & & -23.50 & & & & \\
\hline $13484308+4912271$ & 1.45 & 15.44 & 15.02 & $<120$ & & 0.136 & 5.74 & 1.10 & 1.48 & -53.61 & -15.51 & 3.80 & 3.80 & compact \\
\hline $13491845+1808258$ & 1.07 & 15.53 & 14.94 & $<108$ & & 0.086 & 25.60 & & & -18.50 & & & & regular \\
\hline $14025863+6426228$ & 0.98 & 16.17 & 15.71 & $<99$ & & 0.122 & 14.70 & 1.95 & 5.50 & -28.12 & -26.45 & 3.00 & 2.20 & regular \\
\hline $14080590+5414370$ & 0.82 & 15.89 & 15.56 & $<78$ & & & & & & & & & & tail \\
\hline $14234395+2552515$ & 1.71 & 15.66 & 15.31 & $<162$ & & 0.150 & 7.50 & 1.40 & & -28.80 & & & & compact \\
\hline $14335047+1037077$ & 1.04 & 15.52 & 15.07 & $<117$ & & 0.109 & 28.50 & 4.67 & 6.78 & -26.68 & -10.16 & 2.50 & 5.70 & regular \\
\hline $14343833+3051499$ & 0.95 & 15.77 & 14.86 & $<96$ & & & & & & & & & & double? \\
\hline $14375074-1442498$ & 1.41 & 16.05 & 15.51 & $<114$ & & 0.216 & 5.12 & -0.50 & 1.00 & -29.95 & -12.00 & 2.50 & 7.18 & \\
\hline $14404891-0011195$ & 1.65 & 15.95 & 15.51 & $<138$ & & 0.072 & 16.08 & 3.62 & 6.73 & -22.42 & -16.50 & 1.70 & 3.20 & regular \\
\hline $14445354+2919055$ & 2.97 & 14.46 & 14.07 & $<90$ & & & & & & & & & & double? \\
\hline $14511245+2253010$ & 2.56 & 15.21 & 14.56 & 250 & 330 & 0.119 & 9.90 & & & -36.75 & & & & edge-on \\
\hline $14523534+7354420$ & 1.57 & 15.50 & 14.91 & $<87$ & & 0.176 & 9.08 & 0.90 & 4.30 & -35.03 & -20.00 & 3.10 & & \\
\hline $14535143+2431031$ & 1.33 & 15.82 & 14.94 & $<90$ & & 0.065 & 1.30 & 0.30 & & -27.88 & & & & edge-on \\
\hline $15004675+4803272$ & 2.08 & 15.70 & 15.27 & 290 & 610 & 0.140 & 17.40 & 2.88 & 3.94 & -22.00 & -10.10 & 2.00 & 5.50 & regular \\
\hline $15125997+0724457$ & 2.51 & 15.08 & 14.72 & 210 & & 0.101 & 94.19 & 17.04 & 17.02 & -44.55 & -12.43 & 3.50 & 5.80 & regular \\
\hline $15160785-1608098$ & 1.54 & 15.69 & 14.94 & 210 & 810 & 0.129 & 8.41 & 1.18 & 1.27 & -33.14 & -25.10 & 2.50 & 4.20 & \\
\hline $15163000+5614003$ & 0.81 & 15.97 & 15.81 & $<195$ & & & & & & & & & & compact \\
\hline $15270421+1928206$ & 1.39 & 15.53 & 14.82 & $<132$ & & 0.121 & 2.20 & 0.20 & 0.30 & -54.74 & -13.90 & 2.00 & & companion? \\
\hline $15492435+4822553$ & 0.76 & 15.68 & 15.47 & $<87$ & & 0.072 & 54.60 & 13.60 & 37.60 & -54.65 & -38.38 & 3.60 & 1.40 & compact \\
\hline $15554606+1532218$ & 2.06 & 15.69 & 14.70 & $<111$ & & 0.131 & 33.01 & 8.90 & 19.09 & -66.00 & -43.00 & 1.70 & 2.00 & tail \\
\hline $16025967+5826592$ & 2.01 & 15.35 & 14.95 & 150 & & & & & & & & & & \\
\hline $16053289+2037347$ & 2.45 & 15.55 & 15.17 & $<75$ & & 0.133 & 89.67 & 18.97 & 38.55 & -69.73 & -34.86 & 3.00 & 2.36 & companion? \\
\hline $16132299+4203418$ & 1.99 & 16.29 & 15.12 & 210 & 310 & 0.138 & 13.09 & 3.41 & 8.41 & -64.43 & -34.90 & 2.50 & 3.66 & irregular \\
\hline $16164591+3221543$ & 1.77 & 15.84 & 15.52 & 130 & 430 & 0.103 & 48.00 & 10.96 & 23.50 & -65.00 & -29.33 & 3.80 & 2.40 & regular \\
\hline $16251436+2352427$ & 1.44 & 15.65 & 15.16 & $<123$ & & 0.063 & 18.80 & 3.15 & 7.85 & -27.38 & -18.64 & 2.60 & 3.20 & regular \\
\hline $16275436+5533101$ & 1.17 & 16.07 & 15.46 & $<126$ & & 0.135 & 22.26 & 1.32 & & -33.28 & & & & regular \\
\hline $16452008+4615126$ & 1.12 & 15.66 & 15.28 & $<108$ & & & & & & & & & & compact \\
\hline $17193516+4751517$ & 0.96 & 15.74 & 15.23 & $<147$ & & 0.118 & 15.08 & 2.63 & 3.31 & -26.93 & -17.05 & 4.74 & 5.00 & \\
\hline $17350268+6814020$ & 0.78 & 16.00 & 15.51 & $<63$ & & 0.029 & 1.60 & 0.31 & & -18.34 & & & & \\
\hline $17480224+6723023$ & 1.42 & 15.64 & 15.41 & $<84$ & & & & & & & & & & \\
\hline $17493662+6832182$ & 0.79 & 15.69 & 14.96 & $<87$ & & & & & & & & & & \\
\hline $17512100+5652085$ & 0.96 & 16.17 & 15.67 & $<81$ & & 0.154 & 3.17 & 0.38 & & -33.32 & & & & regular \\
\hline $17533157+5710232$ & 1.50 & 15.30 & 14.75 & $<220$ & & 0.068 & 6.51 & & & -29.86 & & & & companion? \\
\hline
\end{tabular}


M. Haas et al.: Polycyclic aromatic hydrocarbon selected galaxies, Online Material $p 2$

Table 1. continued.

\begin{tabular}{|c|c|c|c|c|c|c|c|c|c|c|c|c|c|c|}
\hline 2MASS & $\begin{array}{c}L W 2 \\
\mathrm{mJy} \text { a) }\end{array}$ & $\begin{array}{c}H \\
\text { mag }\end{array}$ & $\begin{array}{c}K_{s} \\
\mathrm{mag}\end{array}$ & $\begin{array}{r}F_{60} \\
\mathrm{mJy} \text { f) }\end{array}$ & $\begin{array}{l}F_{100} \\
\text { mJy }\end{array}$ & $\overline{z z}$ & $\begin{array}{r}\mathrm{H} \alpha \\
\mathrm{b})\end{array}$ & $\begin{array}{r}\mathrm{H} \beta \\
\mathrm{b})\end{array}$ & $\begin{array}{r}{[\mathrm{OII}]} \\
\mathrm{b})\end{array}$ & $\begin{array}{r}E W \mathrm{H} \alpha \\
\AA \mathrm{c})\end{array}$ & $\begin{array}{r}E W[\mathrm{OII}] \\
\AA \mathrm{c}) \\
\end{array}$ & $\begin{array}{r}E W \mathrm{H} \delta \\
\AA \mathrm{d}) \\
\end{array}$ & $\begin{array}{r}\text { EW Ca II K } \\
\AA \text { d) } \\
\end{array}$ & $\begin{array}{c}\text { Morph } \\
\text { e) }\end{array}$ \\
\hline $17542851+5818226$ & 0.73 & 15.20 & 15.33 & $<98$ & & & & & & & & & & compact \\
\hline $17545857+5121560$ & 1.15 & 15.82 & 15.19 & $<78$ & & 0.071 & 3.50 & 0.94 & & -9.70 & & & & regular \\
\hline $17554655+6638399$ & 1.74 & 15.39 & 15.02 & $<42$ & & 0.043 & 0.22 & 0.03 & & -64.79 & & & & \\
\hline $17562602+5136032$ & 1.38 & 15.42 & 14.95 & $<84$ & & 0.118 & 14.02 & 1.41 & & -65.63 & & & & \\
\hline $17564103+6634162$ & 1.99 & 15.30 & 14.91 & $<33$ & & & & & & & & & & \\
\hline $17571162+6645220$ & 1.22 & 15.94 & 15.67 & $<135$ & & & & & & & & & & \\
\hline $17574141+6648578$ & 1.03 & 15.49 & 15.15 & $<108$ & & & & & & & & & & \\
\hline $17582761+6651113$ & 2.10 & 15.40 & 14.97 & $<45$ & & 0.118 & 14.90 & 2.02 & & -25.87 & & & 3.66 & \\
\hline $18143059+4512303$ & 1.85 & 13.99 & 13.74 & $<74$ & & & & & & & & & & \\
\hline $18233704+6418121$ & 0.86 & 14.94 & 14.60 & $<93$ & & & & & & & & & & regular \\
\hline $18332203+6336009$ & 1.79 & 15.30 & 14.74 & $<90$ & & 0.083 & 28.39 & 4.78 & 5.15 & -63.98 & -35.90 & 4.50 & & regular \\
\hline $19135367+6732331$ & 7.37 & 12.89 & 12.83 & $<90$ & & & & & & & & & & \\
\hline 19184395-3815471 & 1.75 & 15.68 & 14.97 & $<276$ & & 0.160 & & 1.19 & 0.92 & & -44.00 & 2.50 & & \\
\hline $19283755-4124470$ & 1.54 & 14.19 & 14.05 & $<69$ & & & & & & & & & & \\
\hline $20101411-0616586$ & 4.39 & 15.66 & 15.51 & $<150$ & & & & & & & & & & \\
\hline $20285026-1947520$ & 1.05 & 16.10 & 15.24 & $<108$ & & 0.159 & 11.53 & 1.88 & & -17.77 & & & & \\
\hline $20445728-1049227$ & 0.85 & 14.94 & 14.43 & $<75$ & & & & & & & & & & \\
\hline $21582549-3022493$ & 0.82 & 15.94 & 15.38 & $<153$ & & 0.175 & & -0.67 & 0.44 & & -7.57 & 5.06 & 7.21 & \\
\hline $22023161-5657577$ & 2.29 & 15.90 & 15.23 & $<99$ & & 0.083 & 17.43 & 4.44 & 13.57 & -33.50 & -16.65 & 4.54 & 4.80 & \\
\hline $22064236-0353402$ & 2.68 & 15.33 & 14.71 & 180 & 520 & 0.072 & 19.37 & 0.56 & & -30.74 & & & & \\
\hline $22352396-2649402$ & 1.36 & 15.44 & 15.17 & $<108$ & & & & & & & & & & \\
\hline $22485447-5134173$ & 1.42 & 15.76 & 14.89 & 110 & 660 & 0.094 & 14.44 & 2.08 & 9.99 & -44.48 & -30.45 & 2.00 & & \\
\hline $22564496-3637423$ & 1.04 & 15.56 & 14.82 & $<156$ & & & & & & & & & & \\
\hline $22565483-3649515$ & 1.27 & 16.05 & 15.21 & $<156$ & & 0.086 & 6.72 & 1.17 & 3.16 & -29.40 & -15.33 & 4.99 & 3.27 & \\
\hline $22572343-2950575$ & 0.99 & 15.96 & 15.03 & $<90$ & & & & & & & & & & \\
\hline $23032475+1652437$ & 0.82 & 15.99 & 15.06 & $<123$ & & & & & & & & & & \\
\hline $23055422-3539591$ & 1.22 & 15.78 & 14.98 & $<87$ & & 0.162 & 9.13 & 1.16 & 1.60 & -25.69 & -11.34 & 4.27 & 6.46 & \\
\hline $23075850+0537063$ & 1.21 & 15.83 & 15.13 & $<126$ & & 0.136 & 8.50 & 1.90 & & -19.48 & & & & \\
\hline $23080919+0538305$ & 1.47 & 16.01 & 14.82 & $<129$ & & 0.146 & 27.40 & 5.53 & & -21.05 & -12.35 & & & \\
\hline $23094110-2236359$ & 1.58 & 15.03 & 14.39 & & & & & & & & & & & \\
\hline $23134646-6252115$ & 1.11 & 15.46 & 15.30 & $<102$ & & & & & & & & & & \\
\hline $23242107-0555516$ & 3.76 & 15.08 & 14.93 & $<144$ & & & & & & & & & & \\
\hline $23345423+4025496$ & 1.17 & 15.38 & 14.90 & $<140$ & & & & & & & & & & \\
\hline $23370573-0208169$ & 2.25 & 15.61 & 15.12 & $<165$ & & 0.078 & 9.37 & 1.61 & & -67.09 & & & & \\
\hline $23521660+2000553$ & 1.37 & 14.94 & 15.05 & $<111$ & & & & & & & & & & \\
\hline
\end{tabular}

a) LW2 zero magnitude corresponds to $90 \mathrm{Jy}$, i.e. $0.9 \mathrm{mJy}$ corresponds to $12.5 \mathrm{mag}$ (Vega system); b) emission line fluxes as measured in the 1-2" wide slit are given in $10^{-16} \mathrm{erg} / \mathrm{s} / \mathrm{cm}^{2} / \AA$, the uncertainties are $\sim 5-10 \%$; c) negative EW values correspond to emission lines. Mean uncertainties are $\sim 10 \%$; d) mean uncertainties are $\sim 25 \%$; e) morphological appearance in SDSS images. The definitions are: regular, edge-on and irregular sources are extended with $>5^{\prime \prime}$ diameter, regular sources have between face-on and intermediate inclination; compact $=$ regular and $<5^{\prime \prime}$ diameter; tail = irregular, but with bright connected tidal tail(s); double = two equally bright sources; companion = another fainter source nearby $\left(<10^{\prime \prime}\right)$; group $=$ at least two companions; f) two sources are not covered by IRAS; u) ULIRG.

Table 2. Parameters from the Spitzer IRS spectra.

\begin{tabular}{lrrrrrr}
\hline \hline 2MASS & $\begin{array}{r}L W 2 / \\
6-10 \mu \mathrm{m}\end{array}$ & $\begin{array}{r}F 15 \\
\mathrm{mJy}\end{array}$ & $\begin{array}{r}F 24 \\
\mathrm{mJy}\end{array}$ & $\begin{array}{r}F 35 \\
\mathrm{mJy}\end{array}$ & $\begin{array}{rr}{[\mathrm{Ne} \text { III }]} \\
15.55\end{array}$ & $\begin{array}{r}{[\mathrm{S} \mathrm{III}]} \\
18.71\end{array}$ \\
\hline $00285431-7726146$ & 0.57 & 7.03 & 9.3 & 27.2 & 6.1 & 16.0 \\
$00445167-2653191$ & & & 4.8 & 11.7 & - & - \\
$01051501-2612466$ & 0.49 & 2.88 & 4.6 & 11.6 & 5.8 & 12.0 \\
$02542961+1509122$ & 0.52 & 2.15 & 3.0 & 8.1 & 1.6 & 4.2 \\
$03574895-1340458$ & 0.61 & 14.48 & 25.2 & 85.0 & 3.6 & 19.1 \\
$06033357-4509412$ & & & 89.3 & 468.5 & - & - \\
$12482307-0344102$ & & & 4.7 & 12.0 & - & 2.4 \\
$13165999-3336459$ & & & 5.3 & 14.2 & - & 2.6 \\
$13371721+0904430$ & & & 6.2 & 22.8 & - & 2.1 \\
$15160785-1608098$ & & & 3.3 & 9.5 & - & 3.0 \\
$15554606+1532218 *$ & 0.40 & 2.88 & 5.3 & 13.7 & 2.5 & 5.9 \\
$16132299+4203418$ & & & 13.6 & 51.4 & - & 6.1 \\
$19184395-3815471$ & & & 7.7 & 24.8 & - & 0.8 \\
$21573517+0114331$ & 0.42 & 3.06 & 3.9 & 9.6 & $<0.7$ & 3.2 \\
$22023161-5657577$ & 0.58 & 4.34 & 7.6 & 18.7 & $<2.0$ & 8.1 \\
$22565483-3649515$ & 0.58 & 1.60 & 2.2 & 6.0 & $<1.0$ & 5.2 \\
$23055422-3539591$ & & & 3.0 & 7.7 & - & - \\
$23075850+0537063$ & & & 3.2 & 9.5 & - & - \\
$23080919+0538305$ & 0.41 & 3.22 & 4.6 & 11.6 & $<0.7$ & 4.1 \\
\hline
\end{tabular}

The second column gives the fraction of the $6-10 \mu \mathrm{m}$ flux seen in the $L W 2$ passband $(5-8.5 \mu \mathrm{m})$. F15 refers to the flux integrated over the ISOCAM $L W 3$ passband $(12-18 \mu \mathrm{m})$. The uncertainty of the continua F15, F24, and F35 are about 10\%. Emission line fluxes are given in $10^{-15} \mathrm{erg} / \mathrm{s} / \mathrm{cm}^{2}$ with $\sim 10-20 \%$ uncertainties; ${ }^{*}$ ) absolute photometry uncertain, see footnote in Sect. 3.2. 
M. Haas et al.: Polycyclic aromatic hydrocarbon selected galaxies, Online Material p 3

Table 3. Photometry from Spitzer IRAC (3.6-8 $\mu \mathrm{m})$ and MIPS $(24-160 \mu \mathrm{m})$.

\begin{tabular}{lccccccr}
\hline \hline 2MASS & $\begin{array}{r}F 3.6 \\
\mathrm{mJy}\end{array}$ & $\begin{array}{r}F 4.5 \\
\mathrm{mJy}\end{array}$ & $\begin{array}{r}F 5.8 \\
\mathrm{mJy}\end{array}$ & $\begin{array}{r}F 8.0 \\
\mathrm{mJy}\end{array}$ & $\begin{array}{r}F 24 \\
\mathrm{mJy}\end{array}$ & $\begin{array}{r}F 70 \\
\mathrm{mJy}\end{array}$ & $\begin{array}{r}F 160 \\
\mathrm{mJy}\end{array}$ \\
\hline $00465933-2527218^{*}$ & & & & & 5.29 & 53.1 & 158.0 \\
$00482481-2519270 *$ & & & & & 2.52 & 28.5 & 81.0 \\
$00561930-3742185$ & 0.477 & & 0.410 & & 1.50 & 21.5 & 49.9 \\
$13092436+2939351$ & & 0.530 & & 3.09 & & & \\
$13371806-3005123$ & 0.676 & & 0.898 & & 1.57 & 21.4 & \\
$14080590+5414370 *$ & & & & & 6.57 & 55.4 & \\
$15163000+5614003 *$ & 0.335 & 0.309 & 0.290 & 1.61 & 2.75 & 53.9 & 76.3 \\
$17512100+5652085$ & & & & & 2.21 & & \\
$17554655+6638399$ & 0.520 & 0.38 & 0.86 & 2.96 & 4.49 & & \\
$17564103+6634162$ & 1.050 & 0.714 & 0.915 & 4.37 & 4.87 & & \\
$17571162+6645220 *$ & 0.455 & 0.337 & 0.645 & 1.66 & & 34.6 & \\
$17574141+6648578$ & 0.572 & 0.410 & 0.557 & 1.91 & 1.40 & & \\
$17582761+6651113$ & 0.705 & 0.520 & 0.630 & 3.39 & 2.65 & & \\
$17584647+6647585$ & 0.527 & 0.375 & 0.576 & 2.57 & 2.28 & & \\
\hline
\end{tabular}

Uncertainties are about $10 \%$ at $3.6-8 \mu \mathrm{m}$, and less than $30 \%$ at $24-160 \mu \mathrm{m} ;{ }^{*}$ Median redshift of the sample $(z=0.105)$ adopted. 